ص ص ص

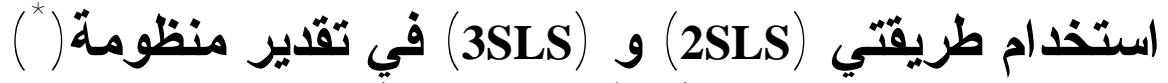

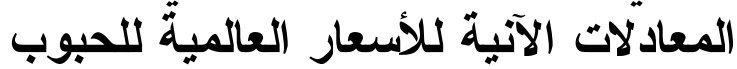

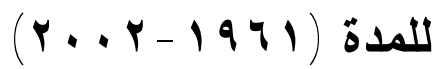

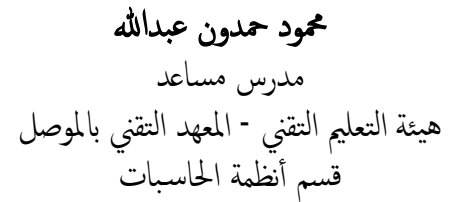

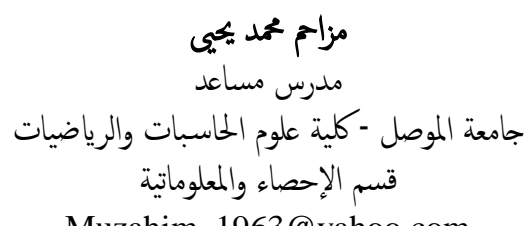

Muzahim_1963@yahoo.com

\begin{abstract}
المستخلص المربعات المبر

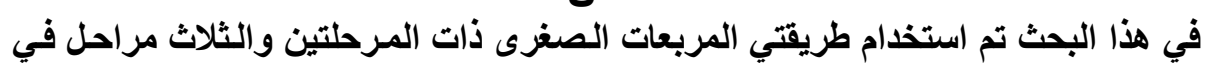

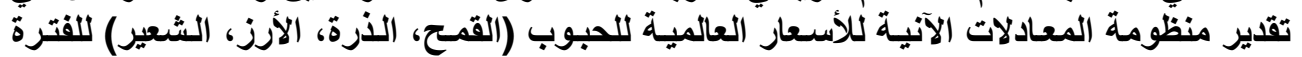

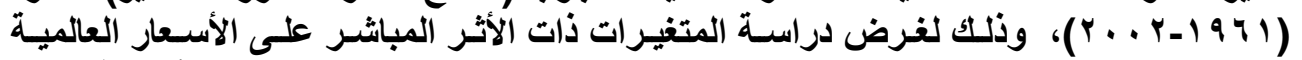

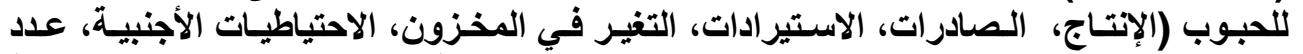

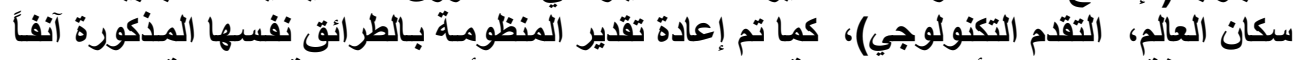

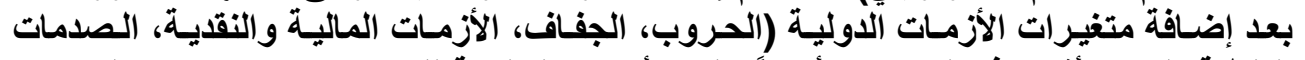

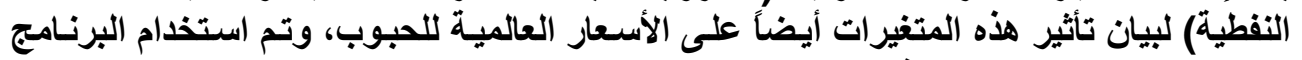

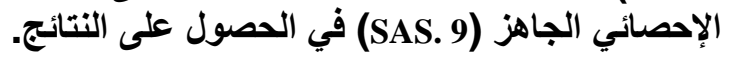

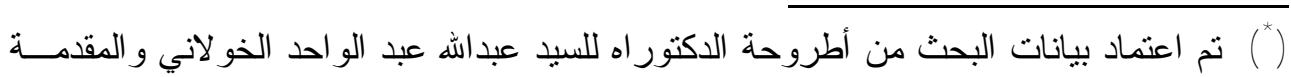

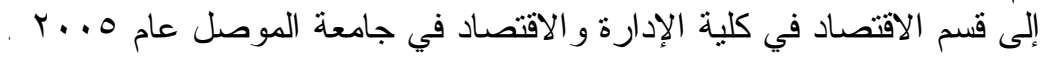

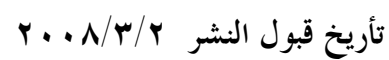

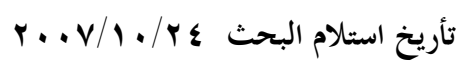




\title{
The Use of (2SLS) and (3SLS) Methods to Estimate the Simultaneous Equations System of the World Prices of the Grains for the Period
} (1961-2002)

\author{
Mozahim M. Yehya \\ Assistant Lecturer \\ Department of Statistics and Informatics \\ University of Mosul
}

\author{
Mahmood H. Abdullah \\ Assistant Lecturer \\ Department of Statistics and Informatics \\ University of Mosul
}

\begin{abstract}
In this paper, (2SLS) \& (3SLS) is used to estimate the simultaneous equation of grains (wheat, maize, rice, barley) for the period (1961-2002). The variables have been considered as direct effects on the world prices of the grains (production, export, import, exchange in the stock, foreigners reserves, the number of the world population, advancement technological). The simultaneous equations have been estimated by the same methods after adding the economic crises to the system (wars, drought, financial and monetary crises and oil shocks) to view the effects also on the world prices of the grains. The (SAS. 9) package is used to obtain the results.
\end{abstract}

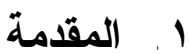

إن بناء النماذج عملية أساسية لمعظم العلوم، سو اء كانت طبيعية أو اجتماعية،

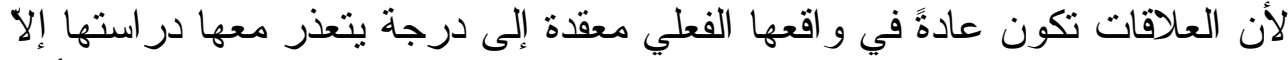

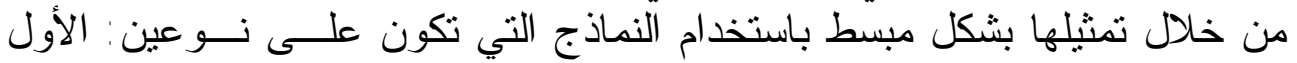

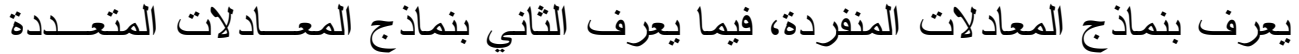

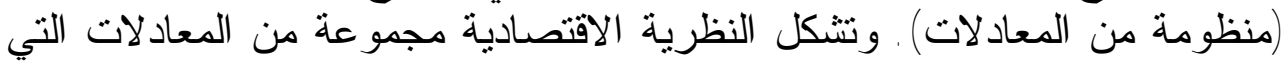

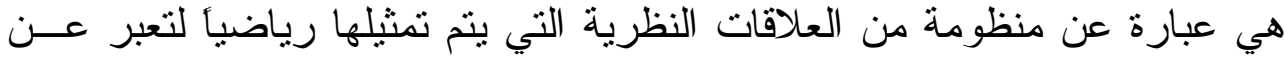

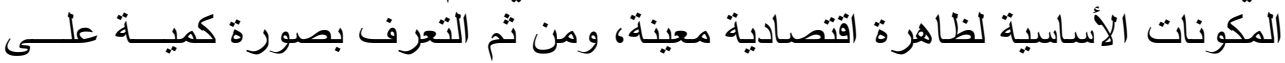

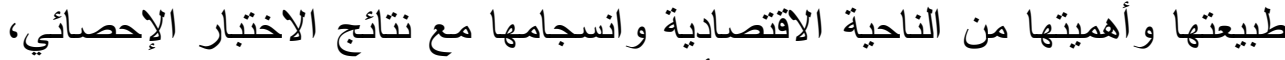

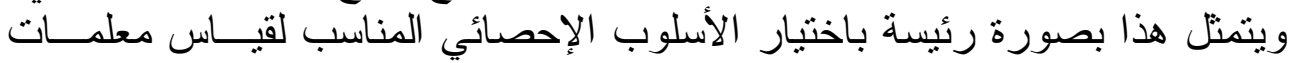

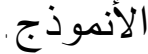

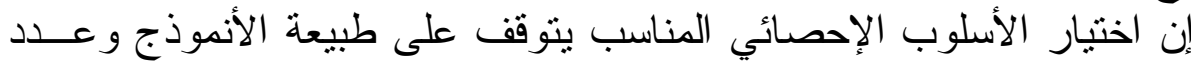

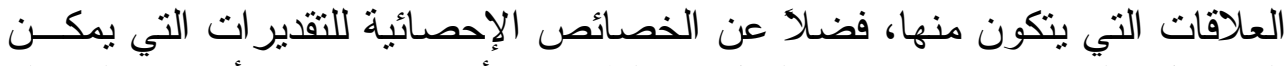

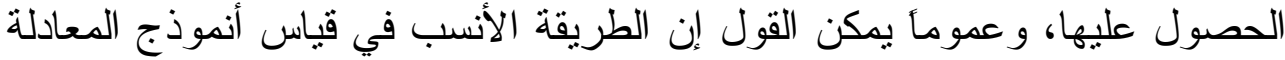

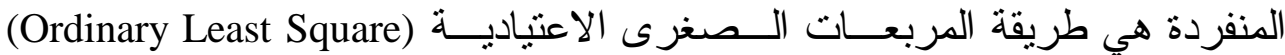

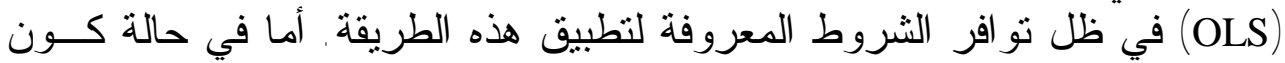

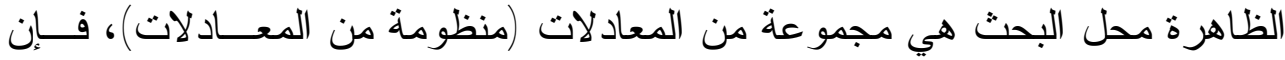

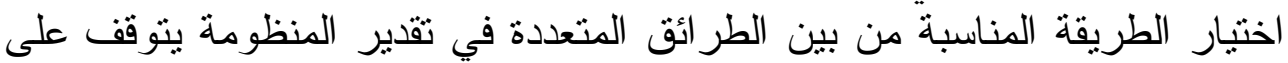

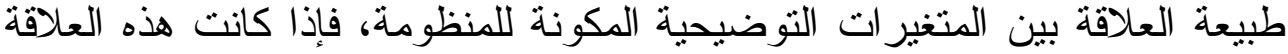

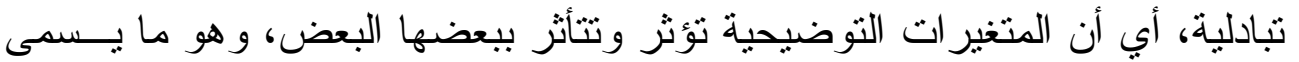
بمنظومة المعادلات الآنية، فإن اختيار الطريقة المناسبة للتقدير يتحدد وفقاً لحالـــة 


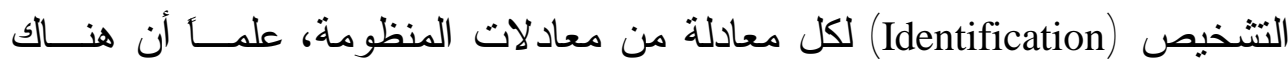

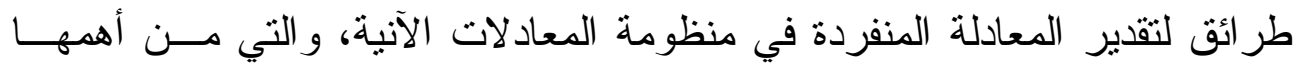

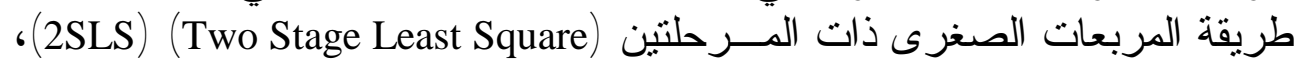

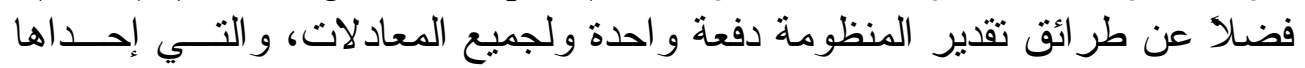

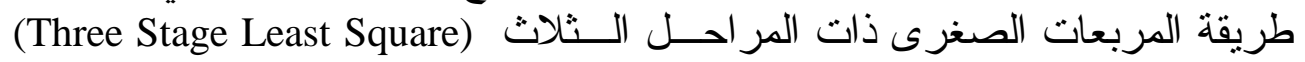

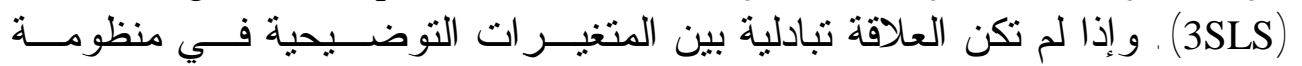

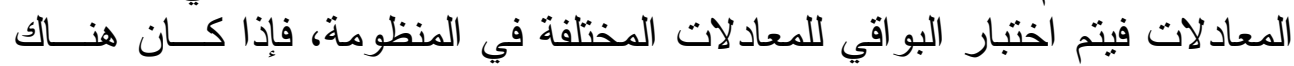

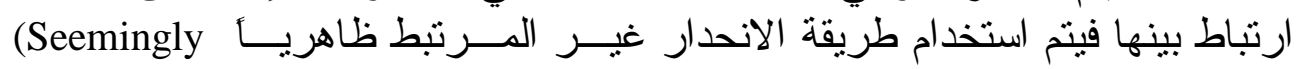
(Unrelated Regression)

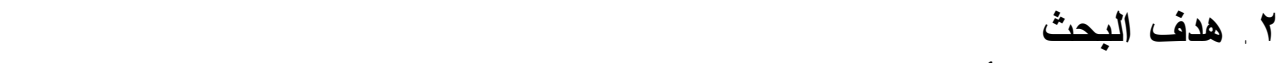

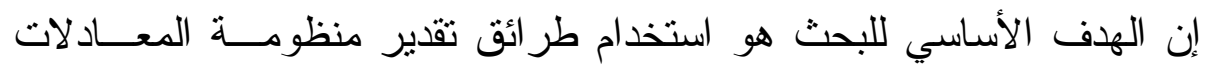

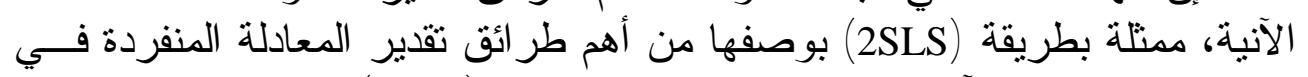

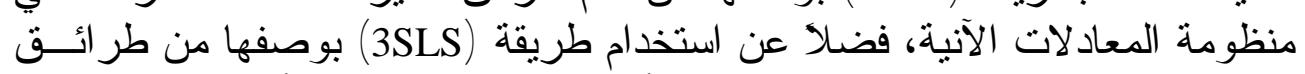

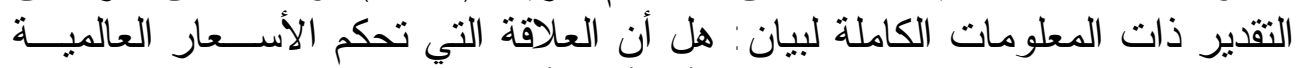

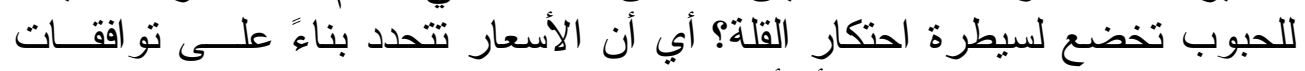

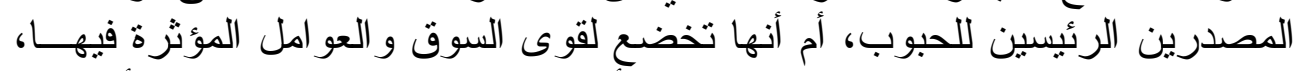

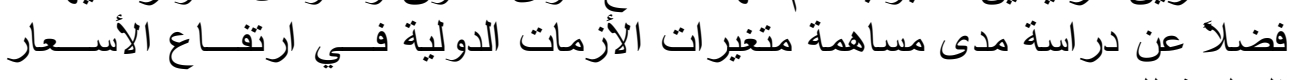

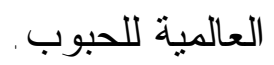

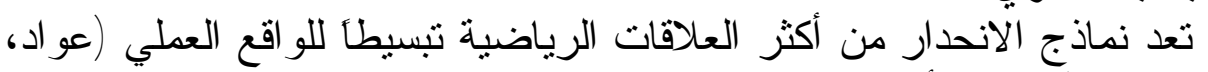

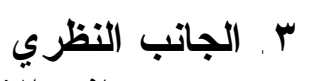

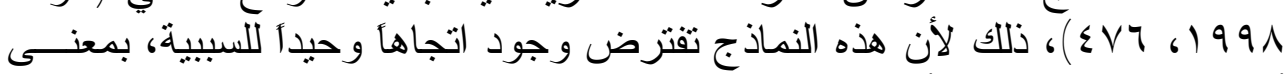

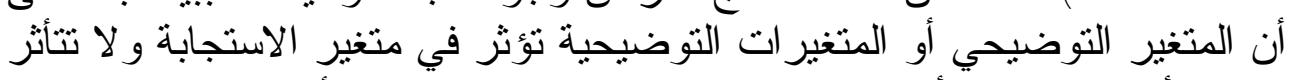

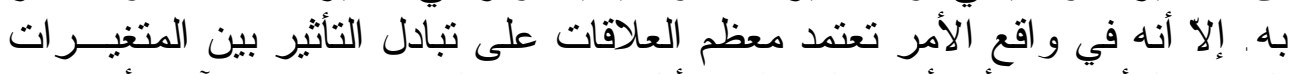

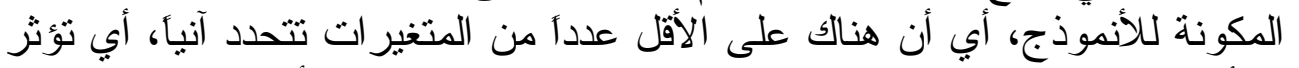

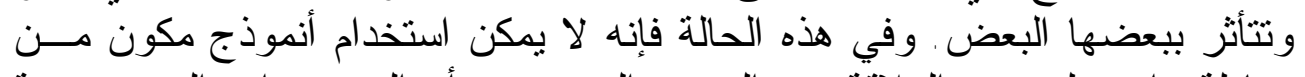

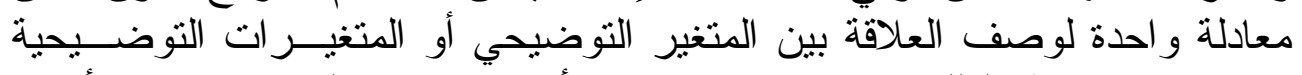

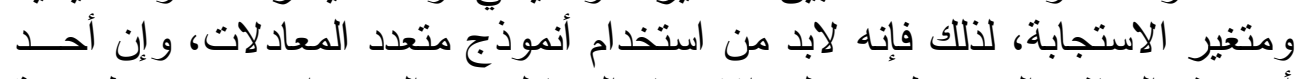

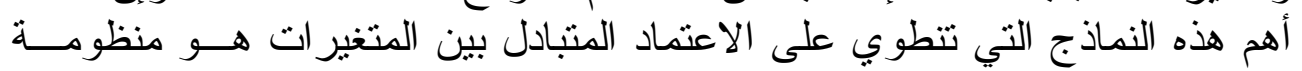

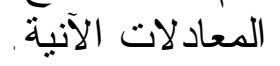

\section{ب - 1 منظومة المعادلات الآنية}

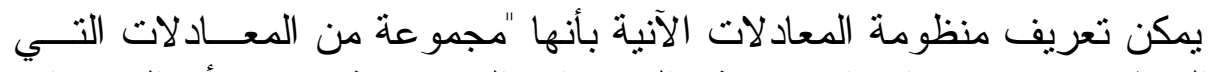

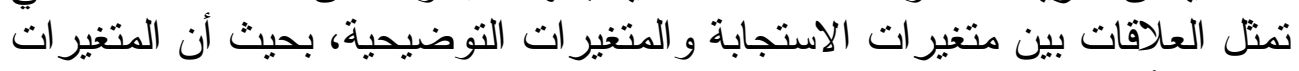

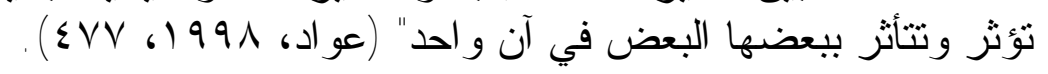




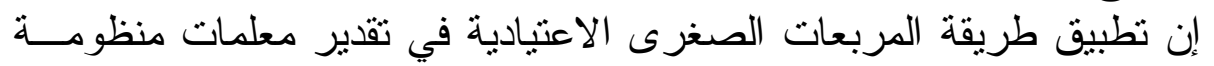

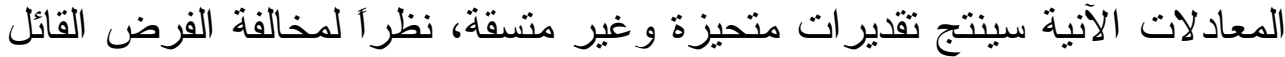

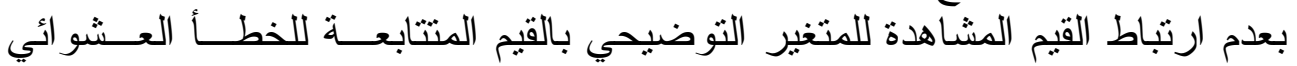

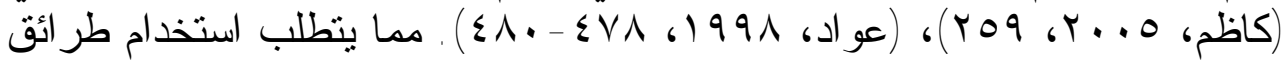

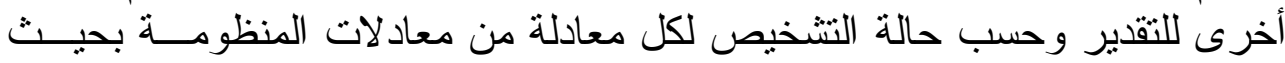
يكون من شأن هذه الطر ائق إعطاء تقدير ات تتمتع بخصائص التصاد التقدير الجيد لمعلمات

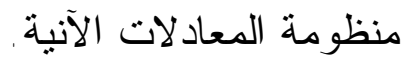

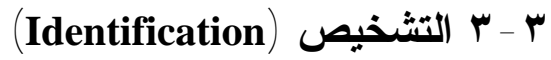

يقصد بالتتخيص اختبار كل معادلة من معادلات المنظومة لمعرفة ما إذا كان

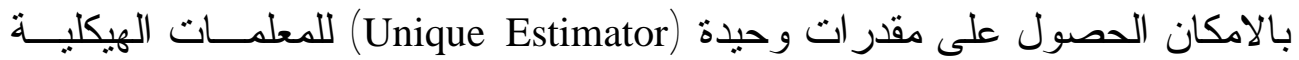

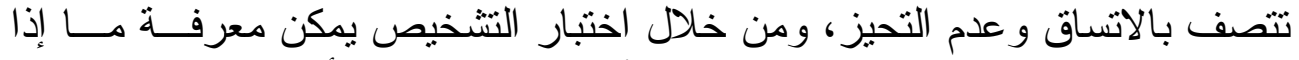

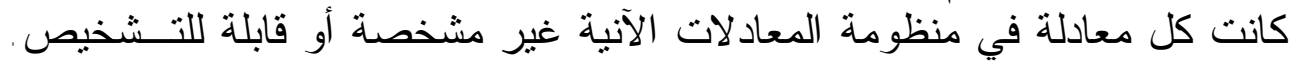

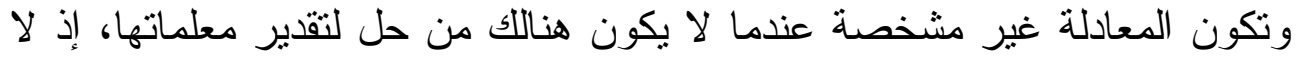

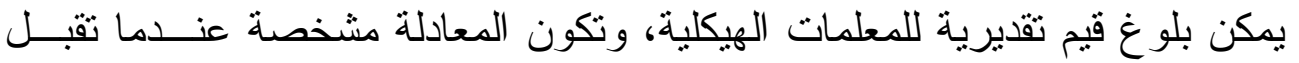

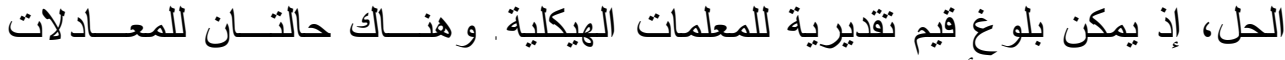

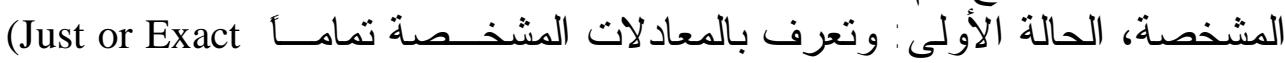

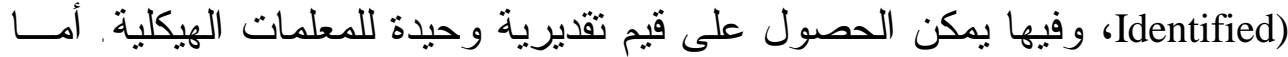

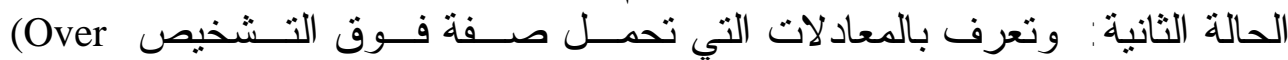

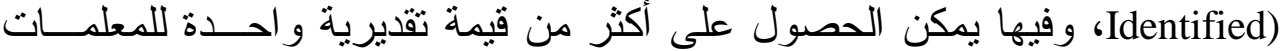

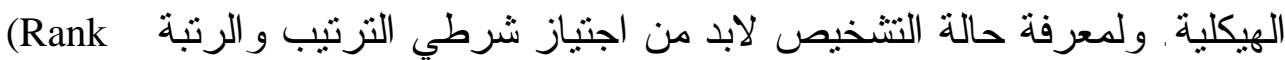
and Order Conditions)

\section{(Order Condition) شرط الترتيب (a}

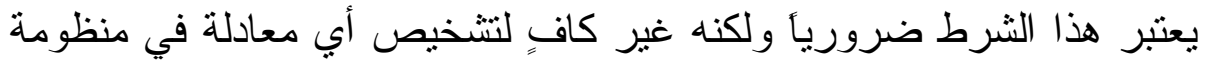

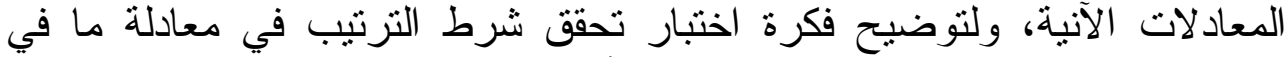

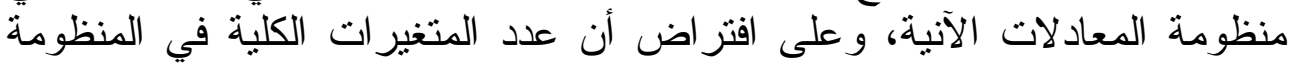

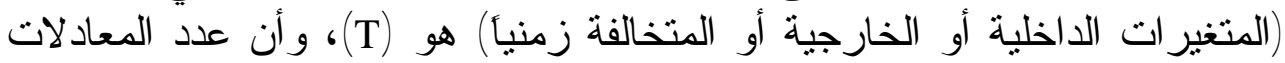

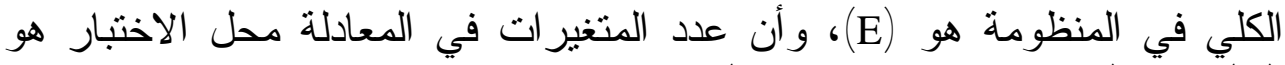
(V)

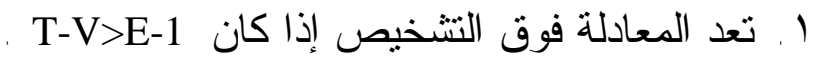

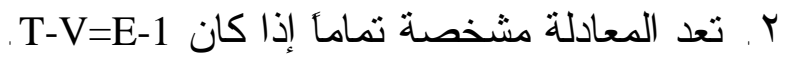
r. بتعد المعادلة تحت التتخيص فيما عدا ذلك. 


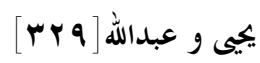

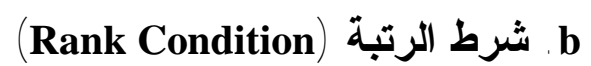

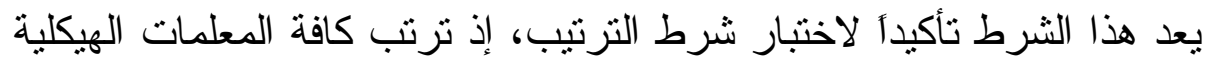

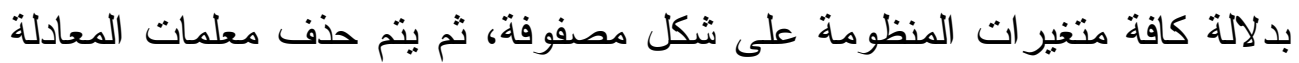

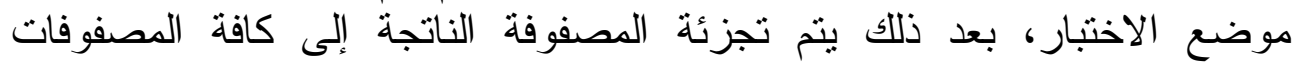

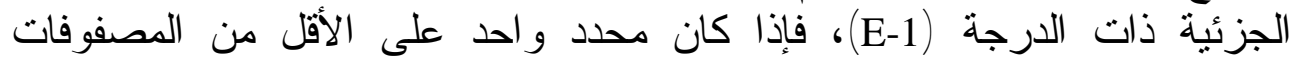

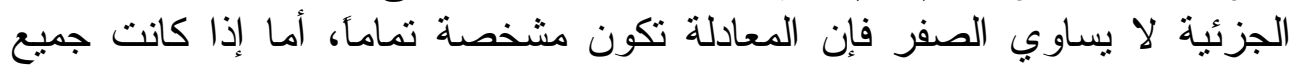
المصفوفات الجزئية ذات الدرجة (E-1) مساوية للصفر فانية الصان المعادلة تكون تحت

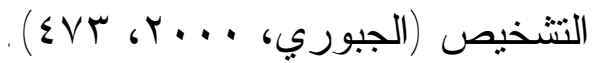

\section{(Two Stage Least Squares) (2SLS)}

$$
\text { r - - طريقة المربعات الصغرى ذات المرحلتين }
$$

تعد طريقة المربعات الصغرى ذات المرحلتين من الطر ائق المهمة في تقدير

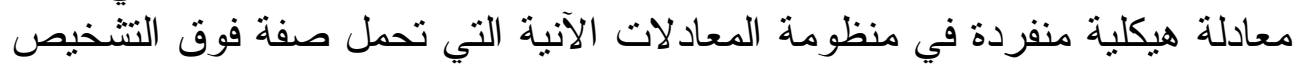

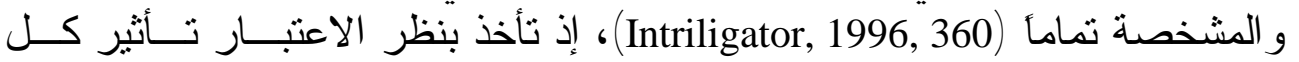

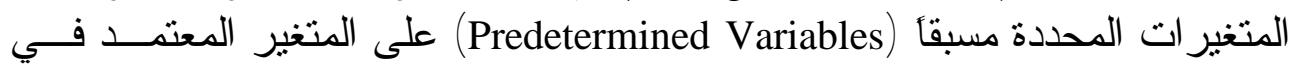

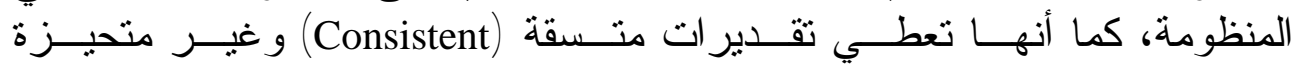

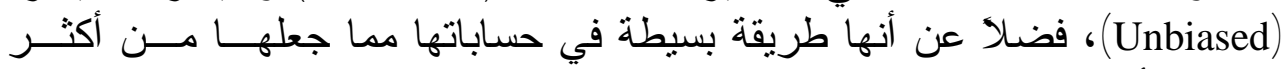

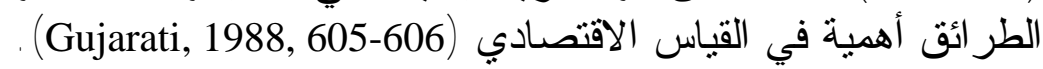

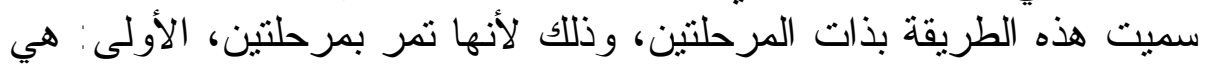

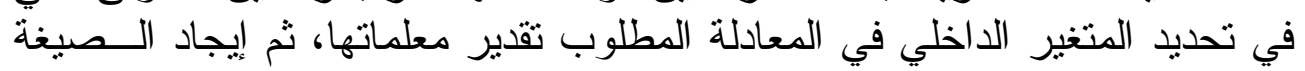

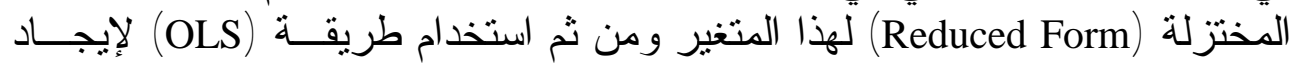

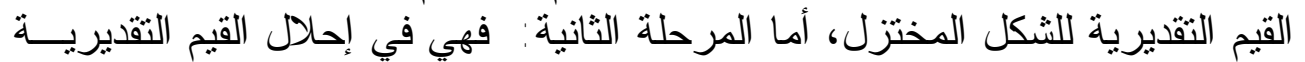

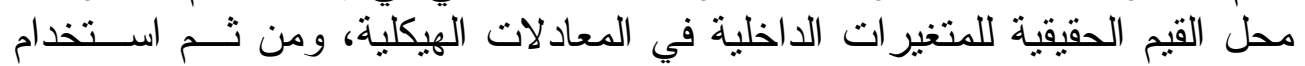

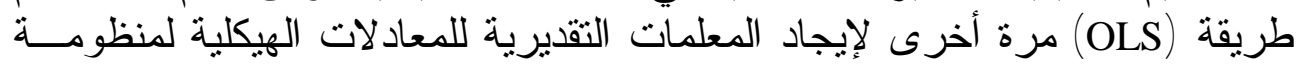
المعادلات الآنية (Intriligator,1996, 361).

كذلك يمكن الوصول إلى مقدرات طريقة (2SLS) باستخدام طريقة المربعات

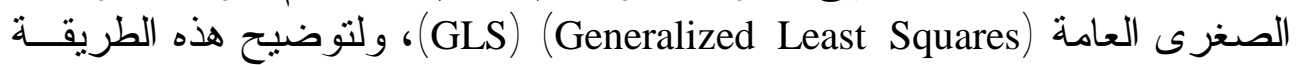

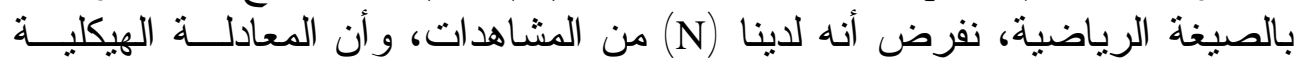
(i) (Structural Equation) $\mathrm{y}_{\mathrm{i}}=\mathrm{Y}_{\mathrm{i}} \gamma_{\mathrm{i}}+\mathrm{X}_{\mathrm{i}} \beta_{\mathrm{i}}+\varepsilon_{\mathrm{i}}$

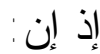

هو : لنجه عمودي لمشاهدات المتغير الداخلي و الذي يمتل متغير الاستجابة في

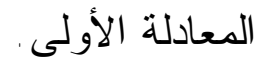




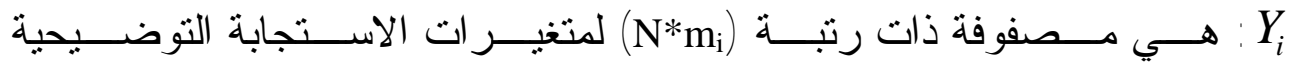
(و هي متغير ات الاستجابة التي تظهر (Explanatory Dependent Variables)

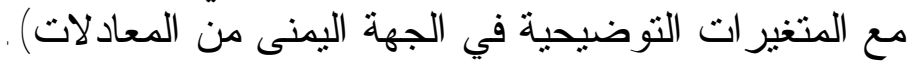

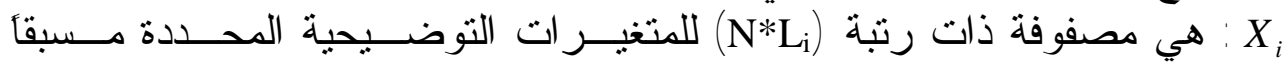
.(Explanatory Predetermined Variables)

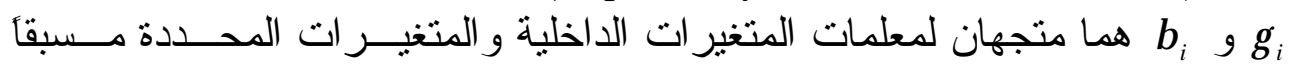
التوضيحية على الترتيب.

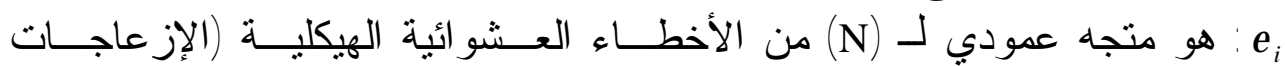
(الاضطر ابات) الهيكلية.

$\mathrm{y}_{\mathrm{i}}=\mathrm{Z}_{\mathrm{i}} \delta_{\mathrm{i}}+\varepsilon_{\mathrm{i}}$

يمكن إعادة كتابة المعادلة الباتية بالصيغة الآتية:

$\mathrm{Z}_{\mathrm{i}}=\left[\begin{array}{ll}\mathrm{Y}_{\mathrm{i}} & \mathrm{X}_{\mathrm{i}}\end{array}\right] \quad ; \delta=\left[\begin{array}{c}\gamma_{\mathrm{i}} \\ \beta_{\mathrm{i}}\end{array}\right]$ بالضرب المسبق للمعادلة ب بالمصفوفة (X') نحصل على:

$\mathrm{X}^{\prime} \mathrm{y}_{\mathrm{i}}=\mathrm{X}^{\prime} \mathrm{Z}_{\mathrm{i}} \delta_{\mathrm{i}}+\mathrm{X}^{\prime} \varepsilon_{\mathrm{i}}$

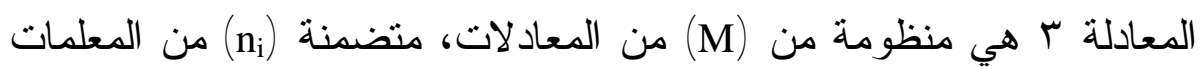

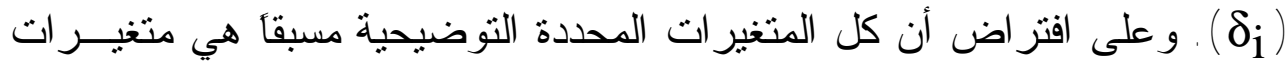

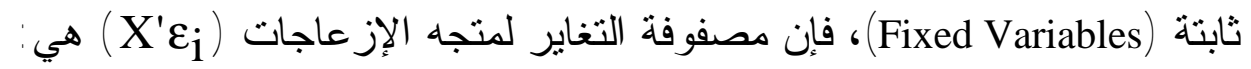

$\mathrm{V}\left(\mathrm{X}^{\prime} \varepsilon_{\mathrm{i}}\right)=\mathrm{E}\left(\mathrm{X}^{\prime} \varepsilon_{\mathrm{i}} \varepsilon_{\mathrm{i}} \mathrm{X}\right)=\sigma_{\mathrm{ii}}\left(\mathrm{X}^{\prime} \mathrm{X}\right)$

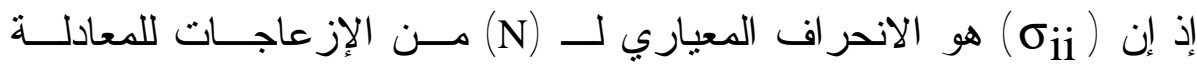

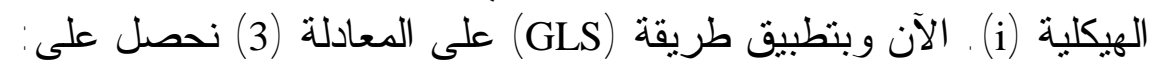

$\mathrm{Z}_{\mathrm{i}}^{\prime} \mathrm{X}\left(\sigma_{\mathrm{ii}} \mathrm{X}^{\prime} \mathrm{X}\right)^{-1} \mathrm{X}^{\prime} \mathrm{y}_{\mathrm{i}}=\mathrm{Z}_{\mathrm{i}}^{\prime} \mathrm{X}\left(\sigma_{\mathrm{ii}} \mathrm{X}^{\prime} \mathrm{X}\right)^{-1} \mathrm{X}^{\prime} \mathrm{Z}_{\mathrm{i}} \delta_{\mathrm{i}}$

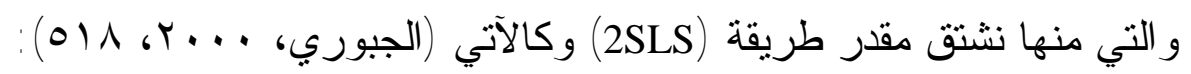




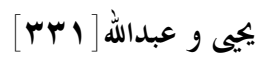

$\hat{\delta}=\left[Z_{i}^{\prime} X\left(\sigma_{i i} X^{\prime} X\right)^{-1} X^{\prime} Z_{i}\right]^{-1} Z_{i}^{\prime} X\left(\sigma_{i i} X^{\prime} X\right)^{-1} X^{\prime} y_{i}$

$\mathrm{V}(\hat{\delta})=\sigma_{\mathrm{ii}}\left[\mathrm{Z}_{\mathrm{i}}^{\prime} \mathrm{X}\left(\mathrm{X}^{\prime} \mathrm{X}\right)^{-1} \mathrm{X}^{\prime} \mathrm{Z}_{\mathrm{i}}\right]^{-1}$

إن مصفوفة التغاير لـ (

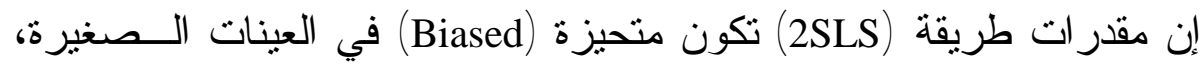

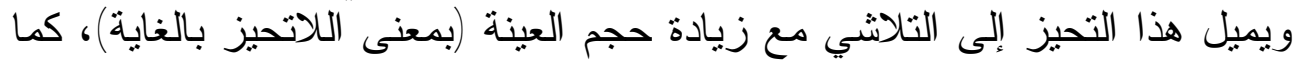
أن مقدر ات هذه الطريقة تتصف بكونها منسقة (Intriligator,1996,PP. (Consistent) .363-368)

\section{ب ــ طريقة المربعات الصغرى ذات المراحل الثلاث (Three Stage Least Squares Method) (3SLS)}

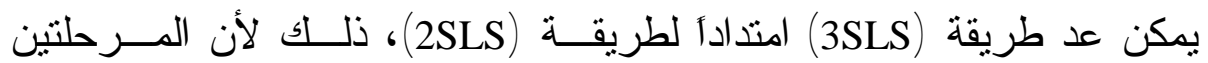

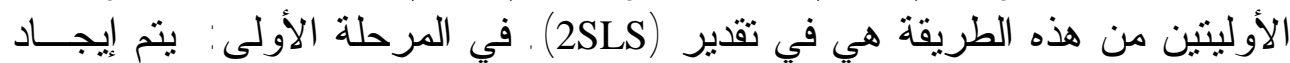

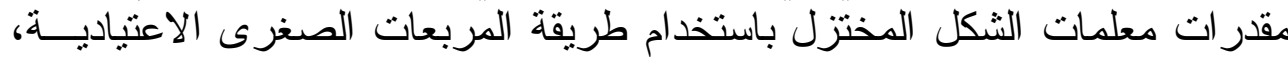

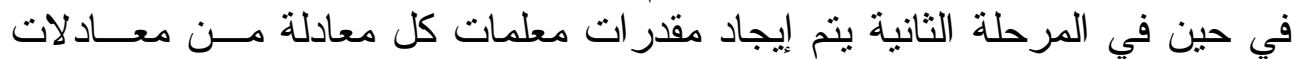

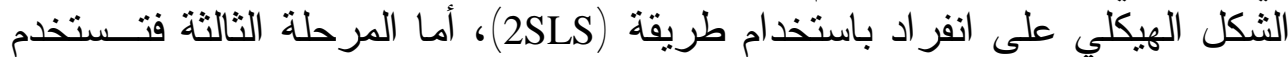

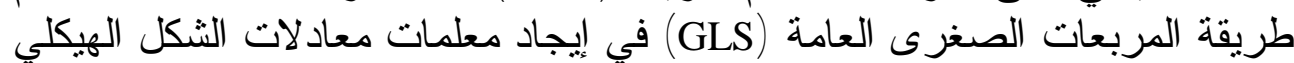

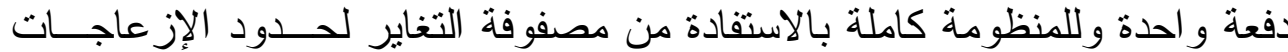
للشكل الهيكلي المقدر من بو اقي المرحلة الثانية (Intriligator,1996, 375).

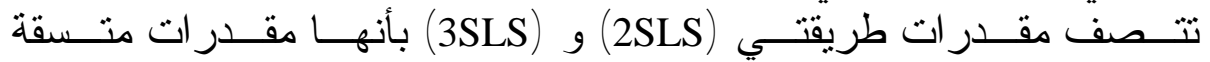

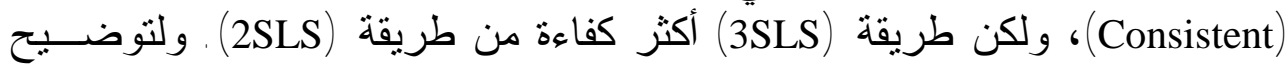

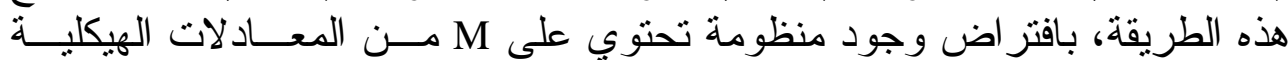

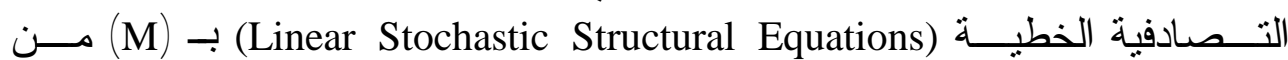

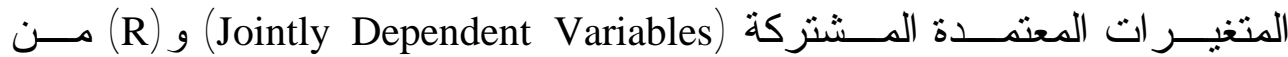

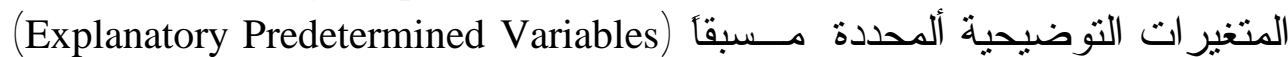

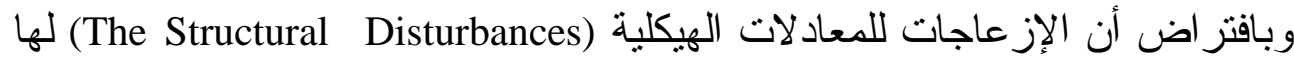

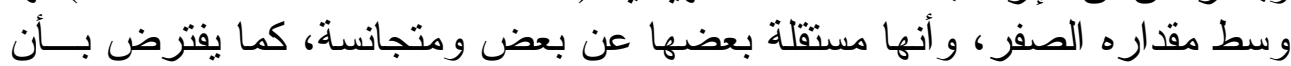

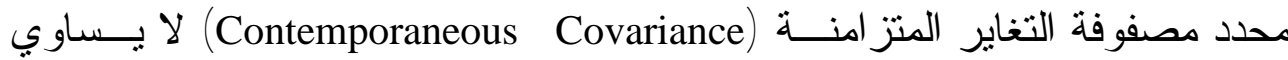

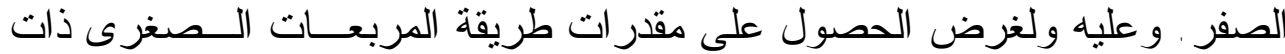

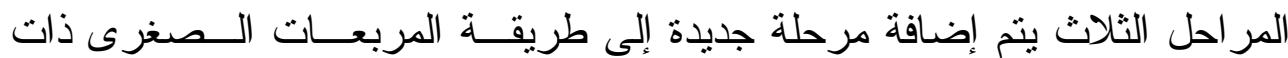

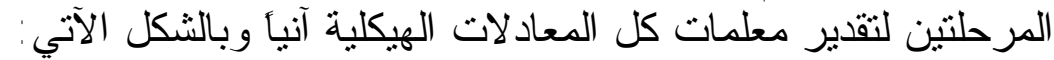
يمكن كتابة المعادلة ب لكل المعادلات مجتمعة بالصيغة الآتية: 


\section{[rre]}

$\left[\begin{array}{l}\mathrm{X}^{\prime} \mathrm{y}_{1} \\ \mathrm{X}^{\prime} \mathrm{y}_{2} \\ \cdot \\ \mathrm{X}^{\prime} \mathrm{y}_{\mathrm{M}}\end{array}\right]=\left[\begin{array}{r}\mathrm{X}^{\prime} \mathrm{Z}_{1} \\ \mathrm{X}^{\prime} \mathrm{Z} \\ \\ \end{array}\right.$

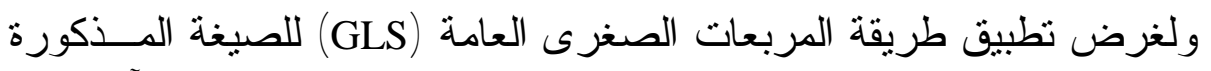
انفا، نحتاج إلى إيجاد مصفوفة التغاير لمتجه البو اقي للمعادلة ^ وبالثكل الآتئي:

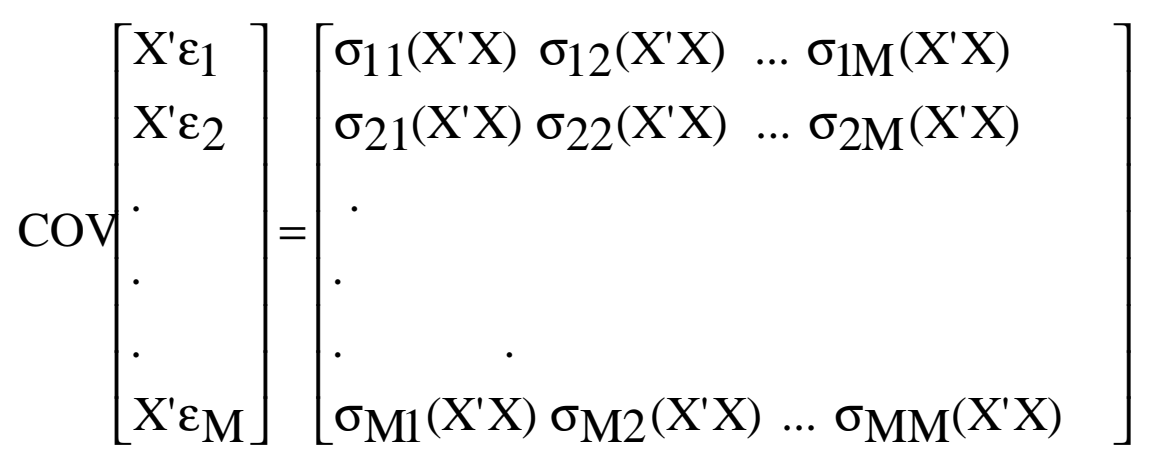

إذ إن (

و'i' كما أن معكوس الصيغة أعلاه هو :

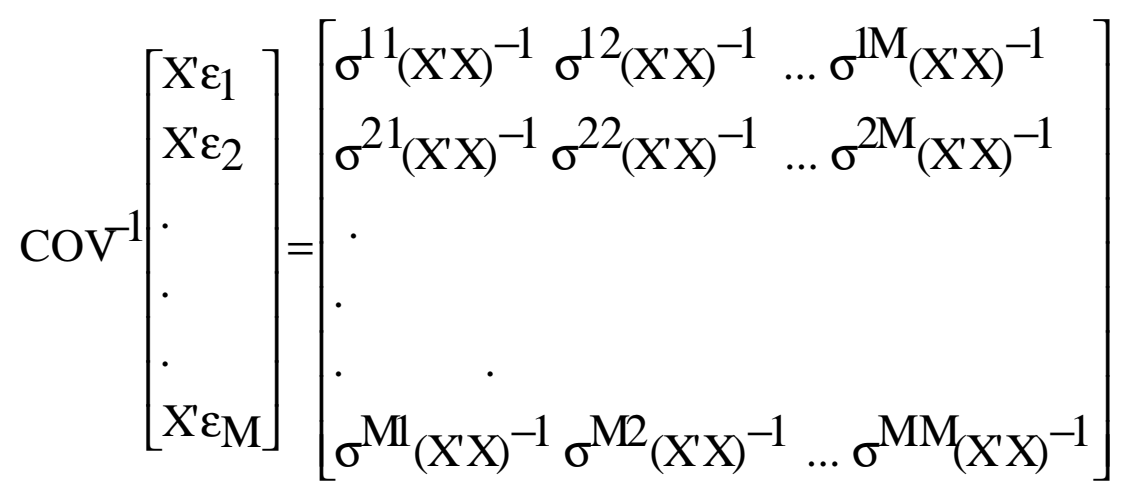




\section{يجيى و عبدالله [بr}

إذ إن ( oii') هو معكوس مصفوفة التغاير المتز امنة للأخطسـاء العـشو ائية

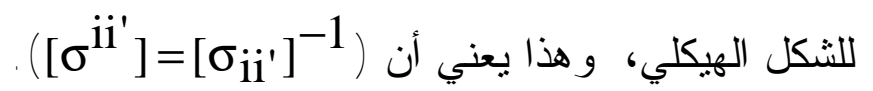

إن النطبيق المباثر لطريقة (GLS) سوف يعطي النتائج الآتية:

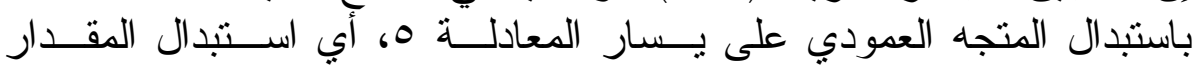
بالمقدار الآتي: $\left(Z_{i}^{\prime} X\left(\sigma_{i i} X^{\prime} X\right)^{-1} X^{\prime} y_{i}\right)$

$$
\begin{aligned}
& {\left[\sigma^{11} Z_{1}^{\prime} X\left(X^{\prime} X\right)^{-1} X^{\prime} y_{1}+\ldots \quad \sigma^{1 M_{Z_{1}^{\prime}}^{\prime} X^{\prime}\left(X^{\prime} X\right)}{ }^{-1} X^{\prime} y_{M}\right.}
\end{aligned}
$$

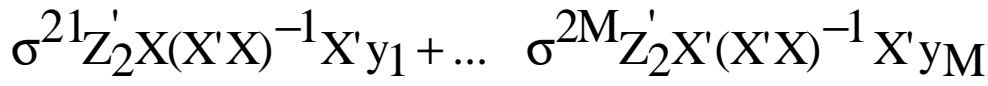

$$
\begin{aligned}
& \sigma^{\mathrm{M} 1} \mathrm{Z}_{\mathrm{M}}^{\prime} \mathrm{X}\left(\mathrm{X}^{\prime} \mathrm{X}\right)^{-1} \mathrm{X}^{\prime} \mathrm{y}_{1}+\ldots \sigma^{\mathrm{MM}} \mathrm{Z}_{\mathrm{M}}^{\prime} \mathrm{X}\left(\mathrm{X}^{\prime} \mathrm{X}\right)^{-1} \mathrm{X}^{\prime} \mathrm{y}_{\mathrm{M}} \\
& \text { بالمقدار الآتي: }\left(Z_{i}^{\prime} X\left(X^{\prime} X\right)^{-1} X^{\prime} Z_{i}\right)
\end{aligned}
$$

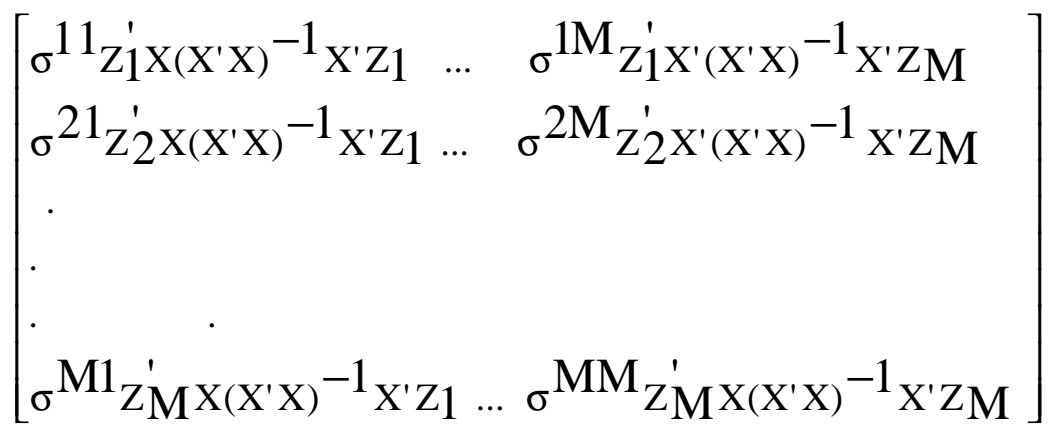

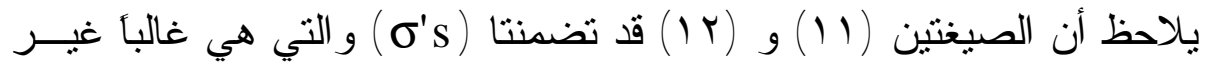

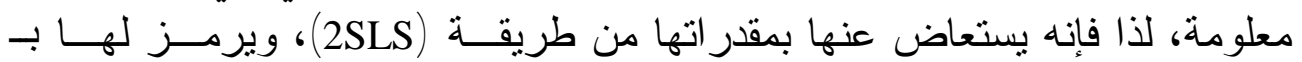

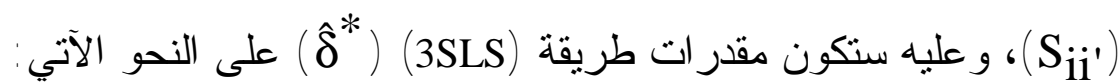




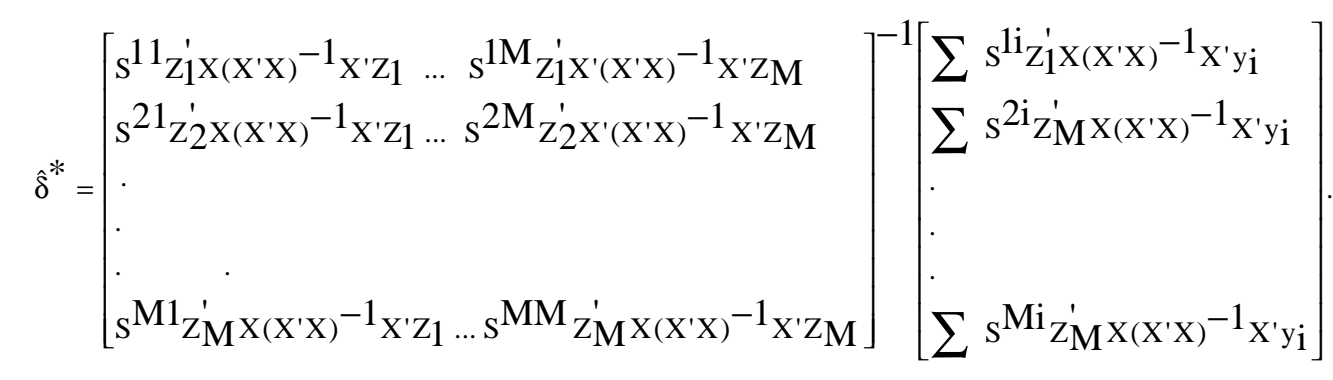

إذ إن مصفوفة التغاير للمقدر ات (

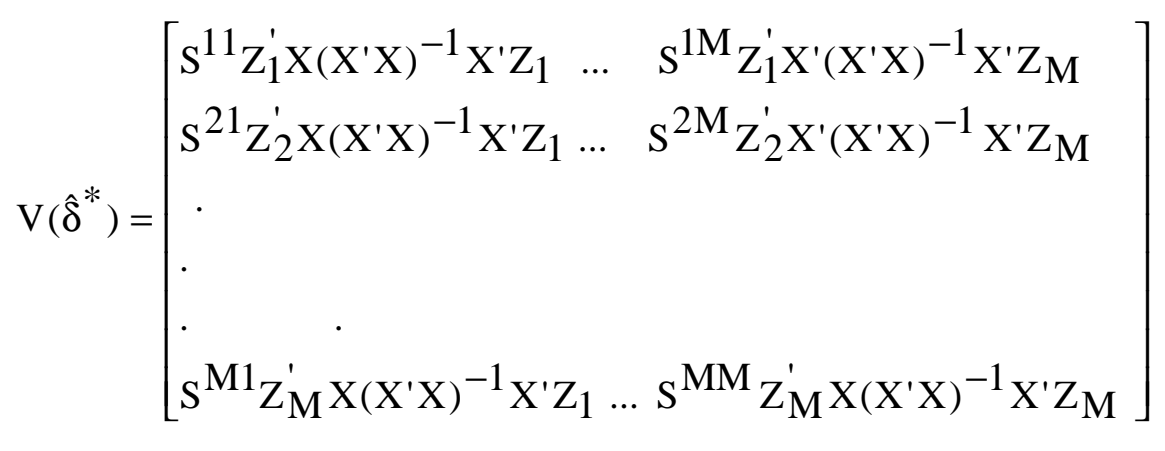

تتصف طريقة (3SLS) بأنها متحيزة ولكنها منسقة، كما أنها أكثر كفاءة مسن

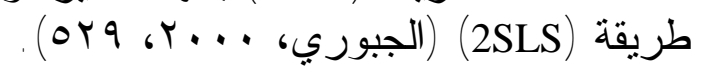

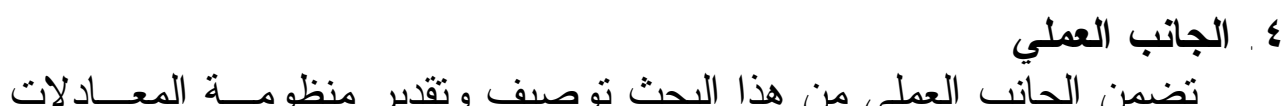

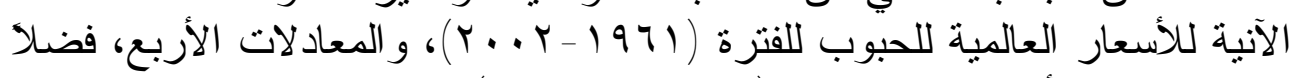

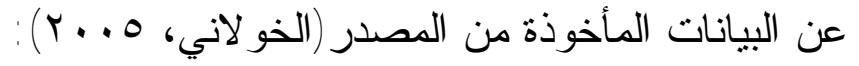

$Y_{1}=a_{0}+a_{1} Y_{2}+a_{2} Y_{3}+a_{3} Y_{4}+a_{4} X_{1}+a_{5} X_{2}+a_{6} X_{3}+a_{7} X_{4}+a_{8} X_{17}+a_{9} X_{18}+a_{10} X_{19}+a_{11} X_{2}$ ${ }_{0}+a_{12} X_{21}+a_{13} X_{22}+a_{14} X_{23}$

$\mathrm{Y}_{2}=\mathrm{b}_{0}+\mathrm{b}_{1} \mathrm{Y}_{1}+\mathrm{b}_{2} \mathrm{Y}_{3}+\mathrm{b}_{3} \mathrm{Y}_{4}+\mathrm{b}_{4} \mathrm{X}_{5}+\mathrm{b}_{5} \mathrm{X}_{6}+\mathrm{b}_{6} \mathrm{X}_{7}+\mathrm{b}_{7} \mathrm{X}_{8}+\mathrm{b}_{8} \mathrm{X}_{17}+\mathrm{b}_{9} \mathrm{X}_{18}+\mathrm{b}_{10} \mathrm{X}_{19}+\mathrm{b}_{11}$ $\mathrm{X}_{20}+\mathrm{b}_{12} \mathrm{X}_{21}+\mathrm{b}_{13} \mathrm{X}_{22}+\mathrm{b}_{14} \mathrm{X}_{23}$

$\mathrm{Y}_{3}=\mathrm{c}_{0}+\mathrm{c}_{1} \mathrm{Y}_{1}+\mathrm{c}_{2} \mathrm{Y}_{2}+\mathrm{c}_{3} \mathrm{Y}_{4}+\mathrm{c}_{4} \mathrm{X}_{9}+\mathrm{c}_{5} \mathrm{X}_{10}+\mathrm{c}_{6} \mathrm{X}_{11}+\mathrm{c}_{7} \mathrm{X}_{12}+\mathrm{c}_{8} \mathrm{X}_{17}+\mathrm{c}_{9} \mathrm{X}_{18}+\mathrm{c}_{10} \mathrm{X}_{19}+\mathrm{c}_{11}$ $\mathrm{X}_{20}+\mathrm{c}_{12} \mathrm{X}_{21}+\mathrm{c}_{13} \mathrm{X}_{22}+\mathrm{c}_{14} \mathrm{X}_{23}$

$\mathrm{Y}_{4}=\mathrm{d}_{0}+\mathrm{d}_{1} \mathrm{Y}_{1}+\mathrm{d}_{2} \mathrm{Y}_{2}+\mathrm{d}_{3} \mathrm{Y}_{3}+\mathrm{d}_{4} \mathrm{X}_{13}+\mathrm{d}_{5} \mathrm{X}_{14}+\mathrm{d}_{6} \mathrm{X}_{15}+\mathrm{d}_{7} \mathrm{X}_{16}+\mathrm{d}_{8} \mathrm{X}_{17}+\mathrm{d}_{9} \mathrm{X}_{18}+\mathrm{d}_{10} \mathrm{X}_{19}+$ $d_{11} X_{20}+d_{12} X_{21}+d_{13} X_{22}+d_{14} X_{23}$ 


$$
\text { Y Y2 }
$$

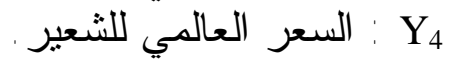

X الصادر X

Xـ : التغير في المخزون العالمي : X

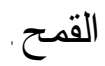

X

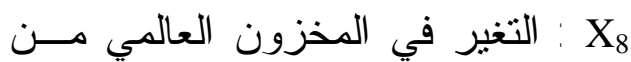

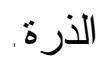

X الصادر X

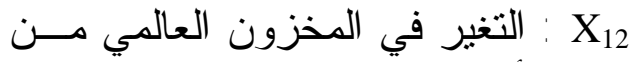

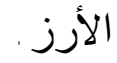

X

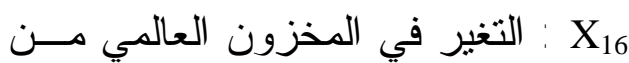

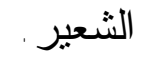

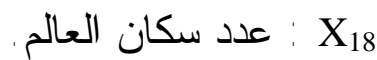

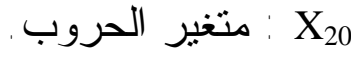

Xتغير الأزمات المالية و النقدية.

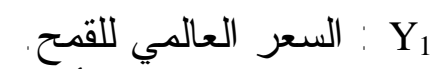

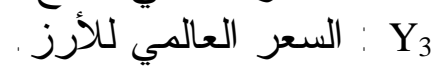

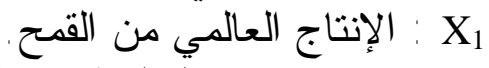
X الاستير ادات العالمية من القمح. X X

X X X7 : الاستير ادات الّعالمية من الذرة. X X الإنتاج العالمي من الأرز . X9 X X X الاستير ادات العالمية من الأرز . X X . الإنتاج العالمي من الثعير X

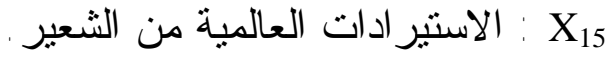
X : متغير الزمن المعبر عن مستوى : X

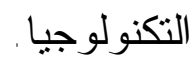

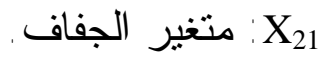
X

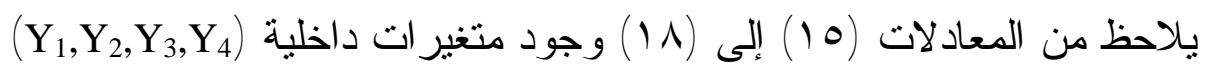

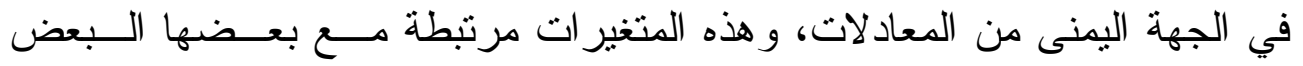

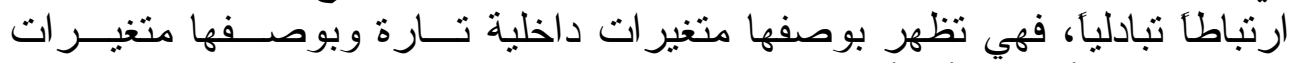

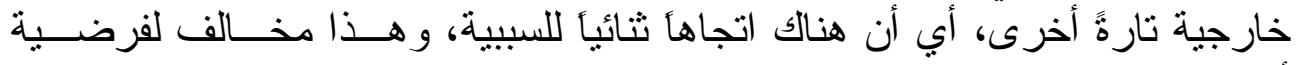

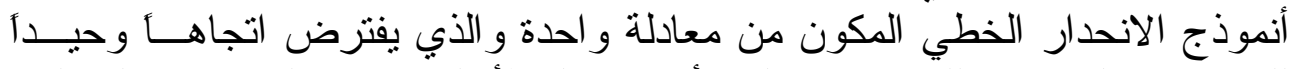

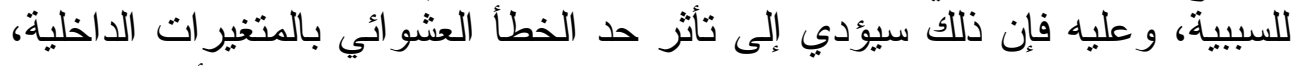

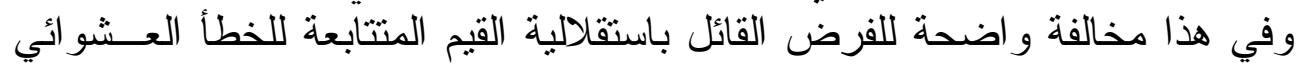
عن القيم المشاهدة للمتغير ات التوضيحة لإنية.

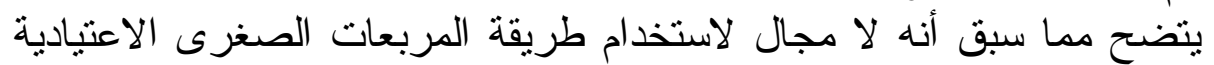

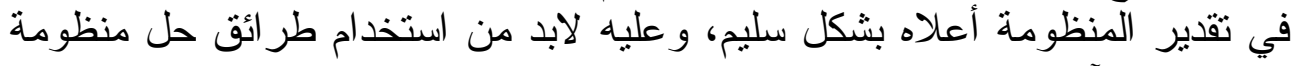

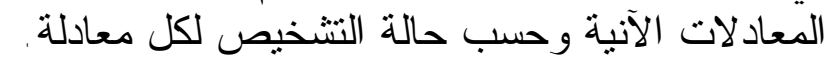

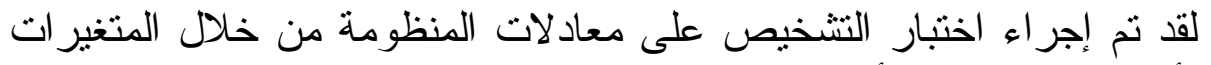

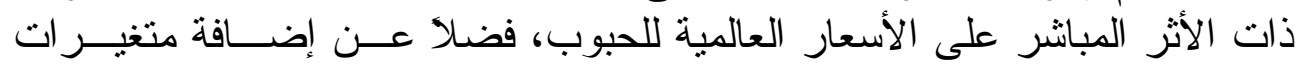

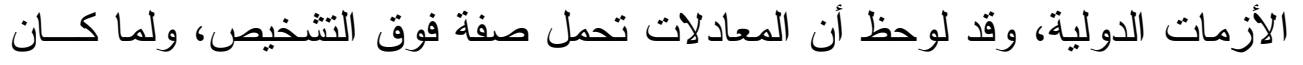


البحث يؤكد أهمية تحقيق أفضل تعبير قياسي للعلاقة الاقتصادية قيد الدرس بحيث

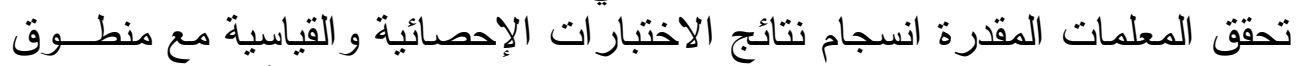

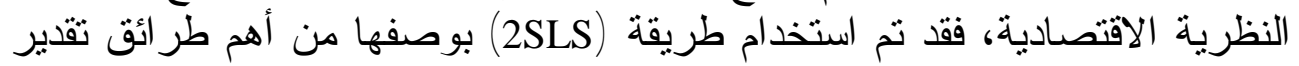

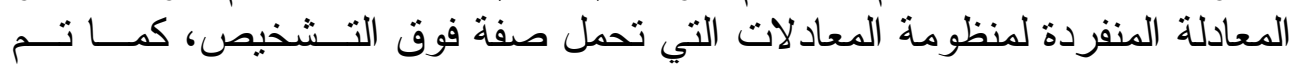

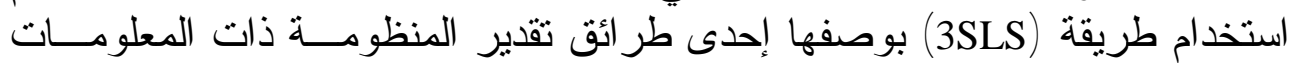

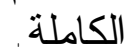

ع - 1 مناقشة النتائج

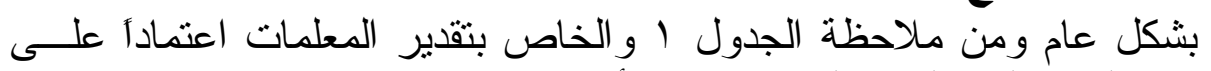

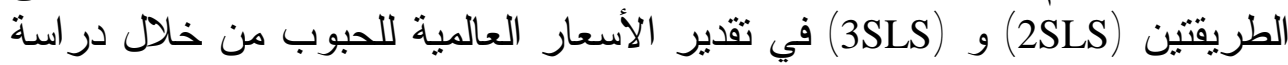

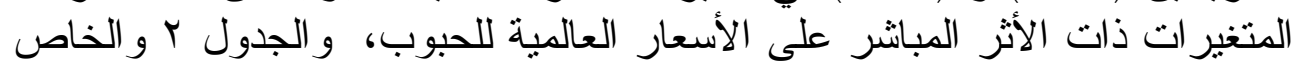

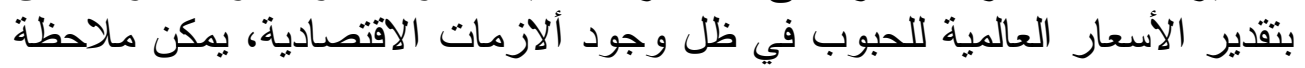

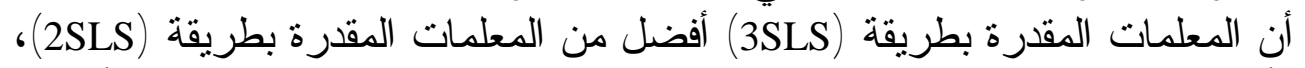

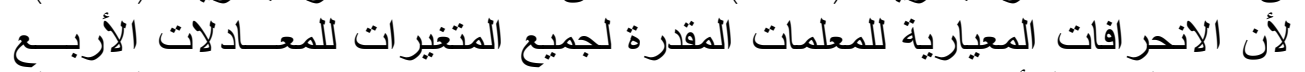

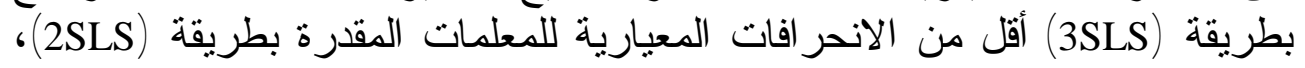

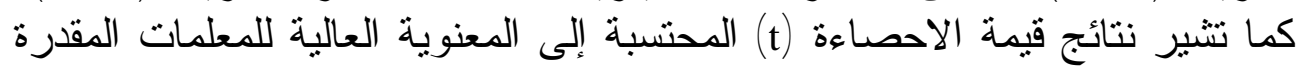

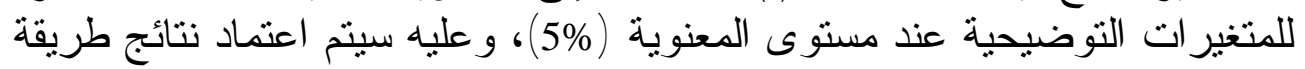
(3SLS) وفيماً يأتي خلاصة لنتائج (3SLS) التي انبتقت عن كل معادلة من معسادلات

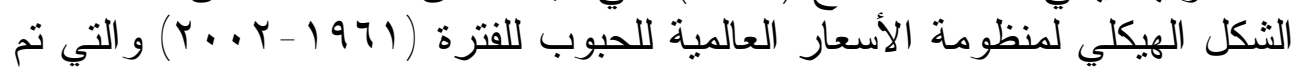

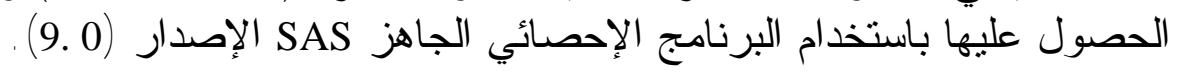

a a a السعر العالمي للقمح

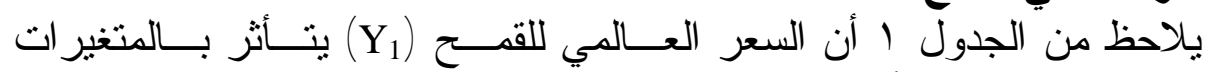
(Y, $\left.\mathrm{Y}_{2}, \mathrm{Y}_{3}, \mathrm{X}_{3}\right)$

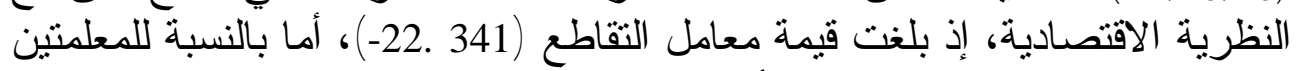

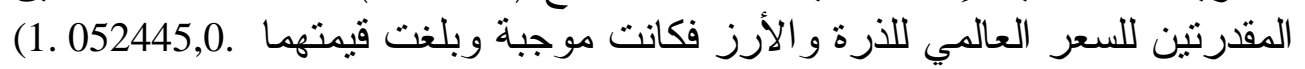

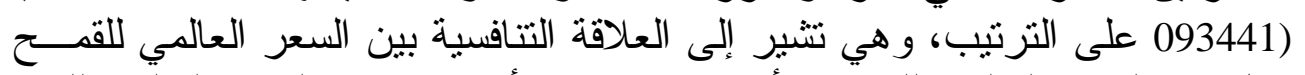

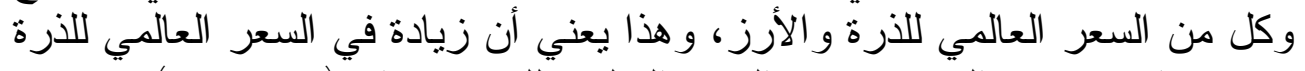

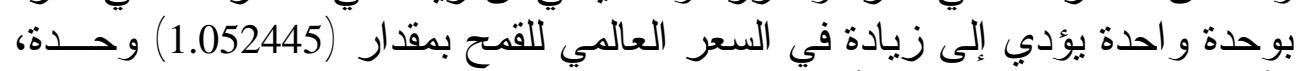

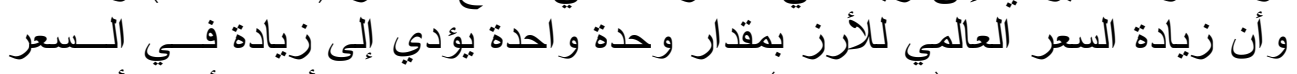

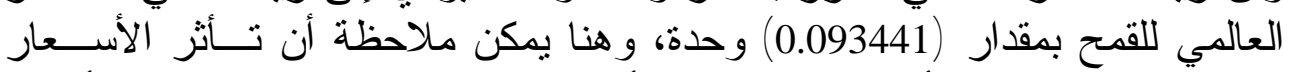

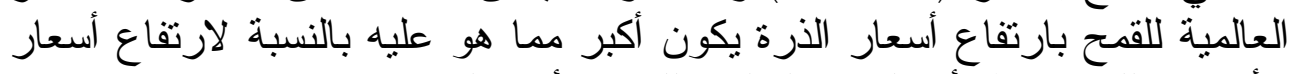

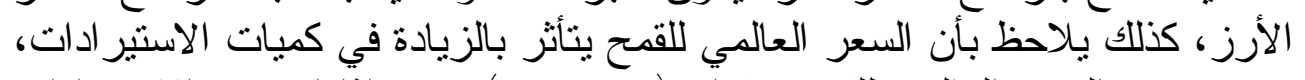

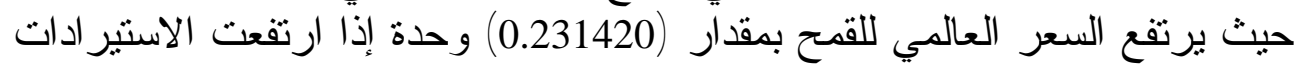

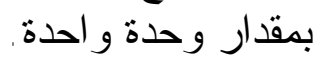




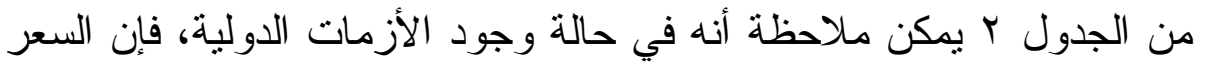

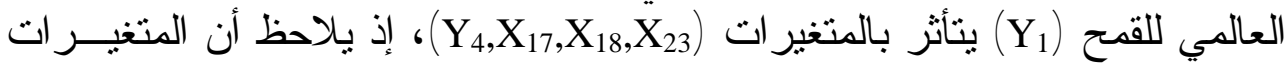

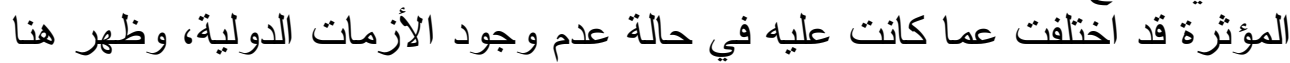

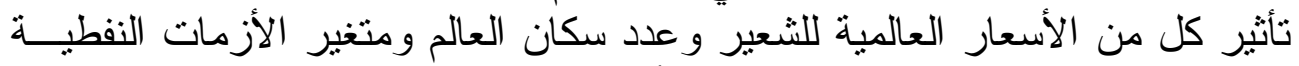

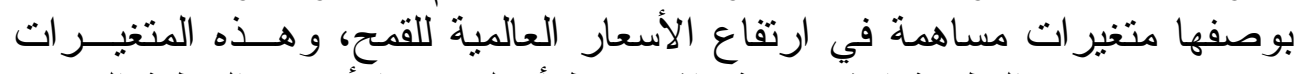

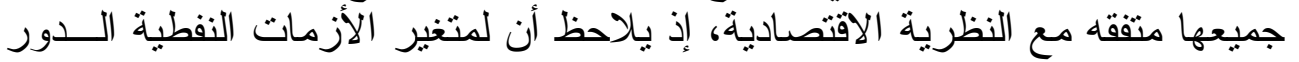

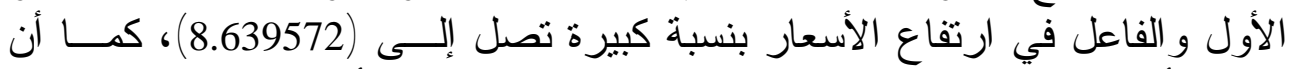

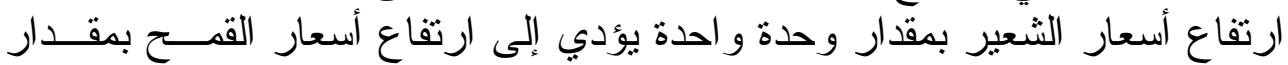

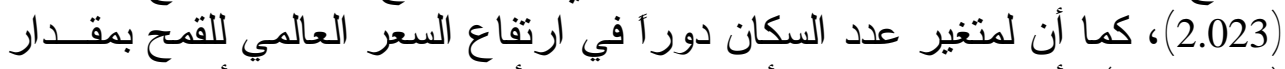

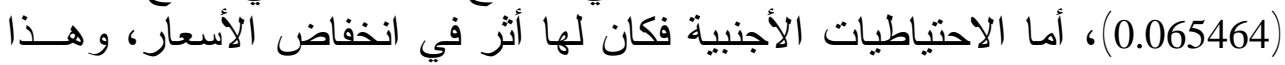

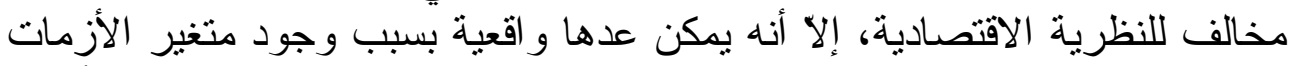

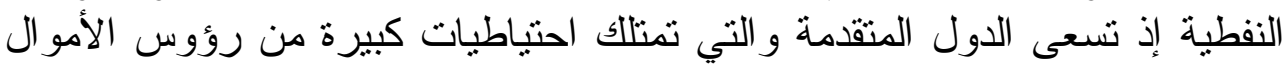

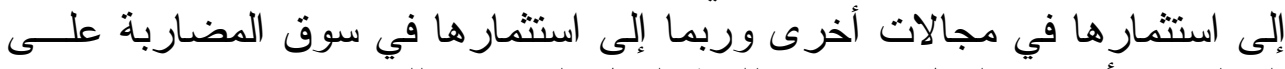

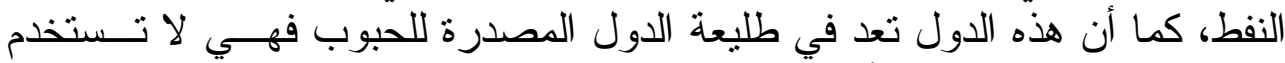

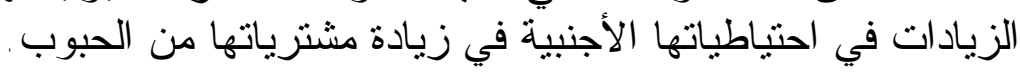

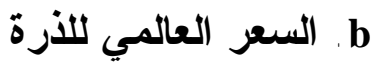

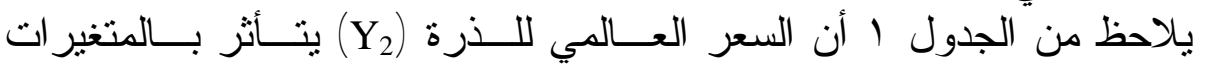

(Y, $\left.{ }_{3}, Y_{4}, X_{7}\right)$

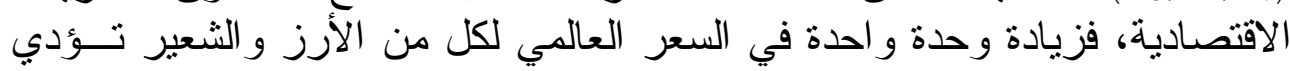

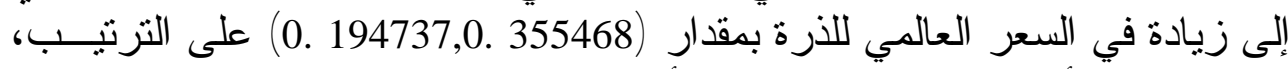

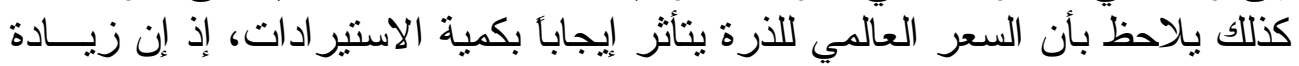

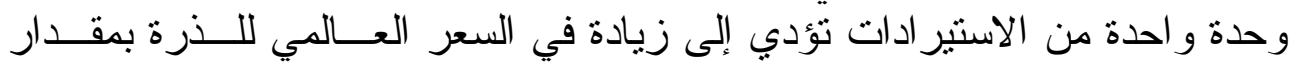

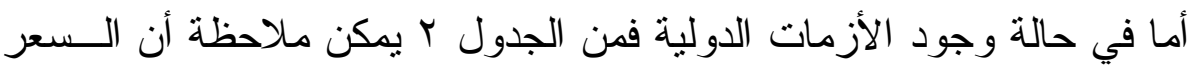

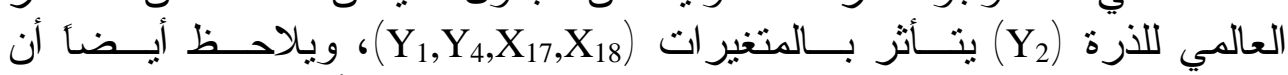

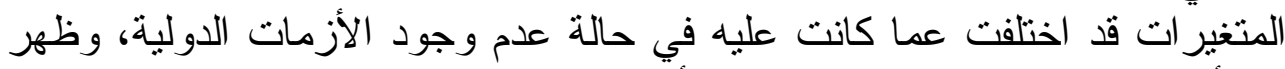

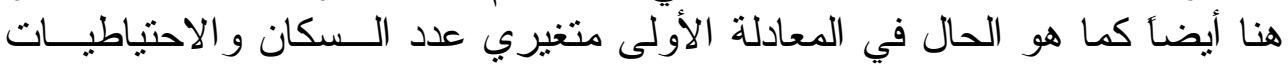

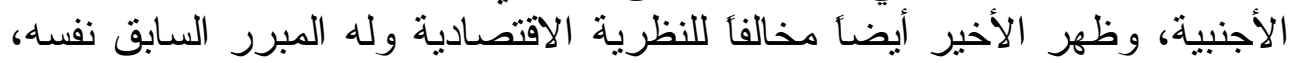

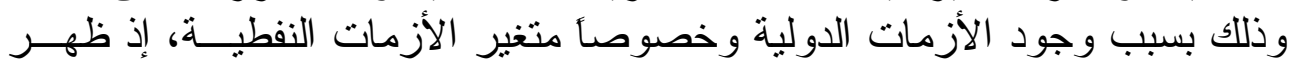

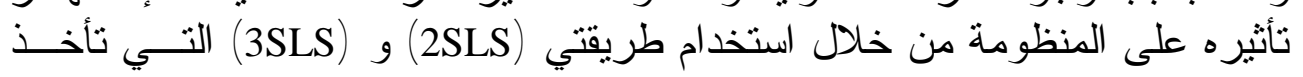

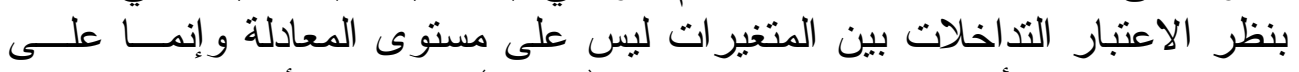

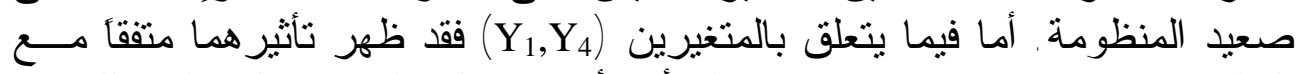

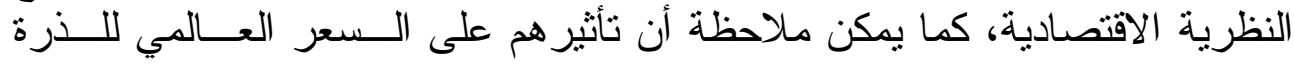

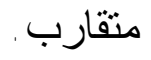




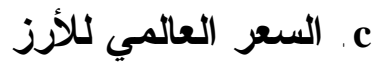

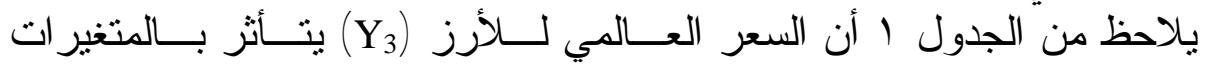
التوضيحية (Y,

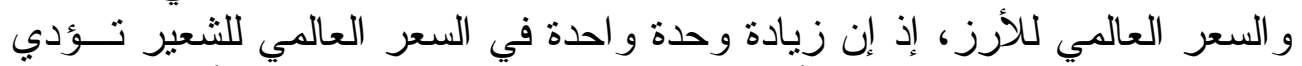

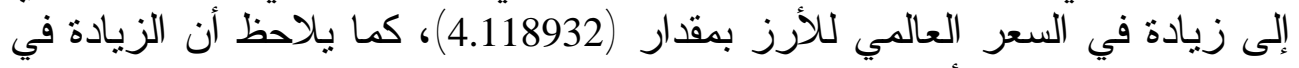

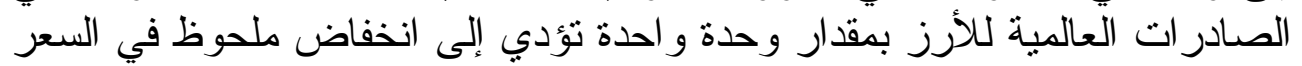

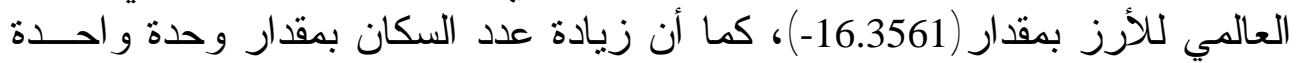

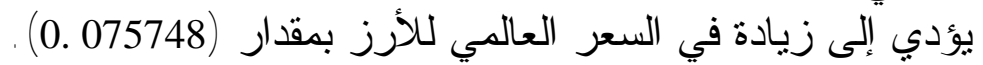

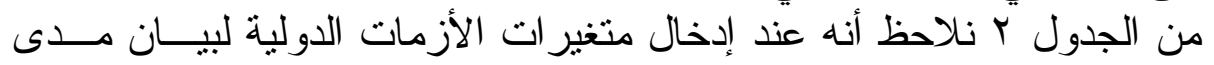

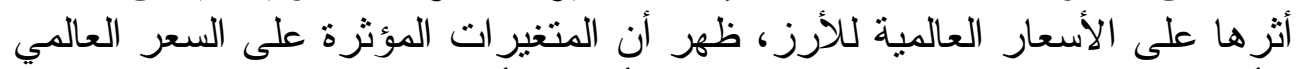

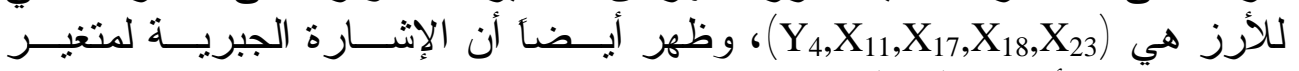

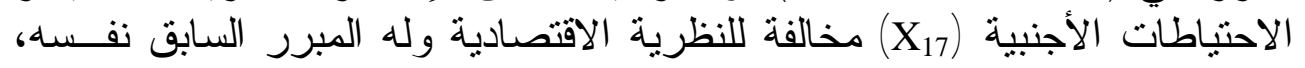

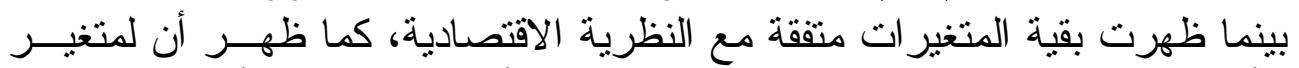

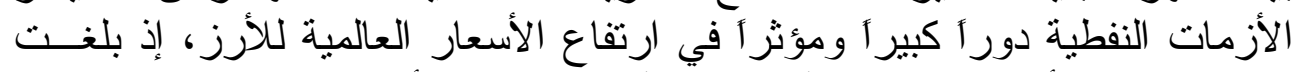

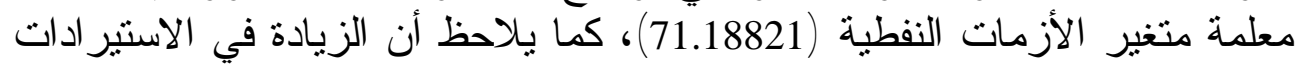

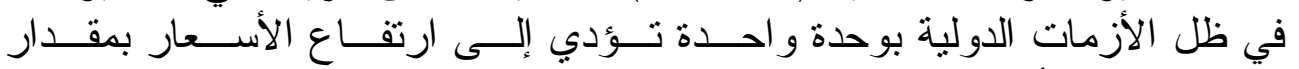

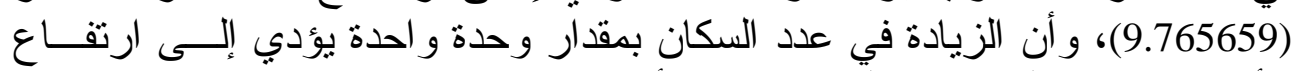

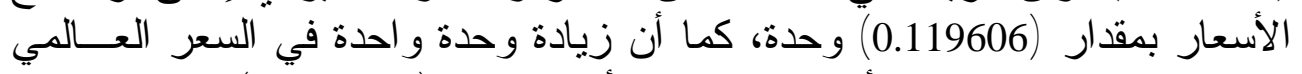

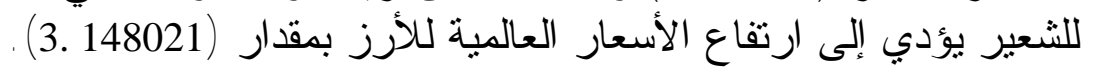

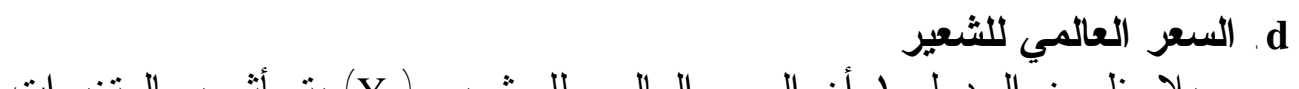

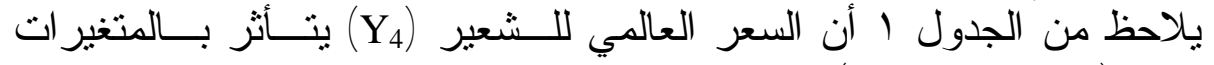

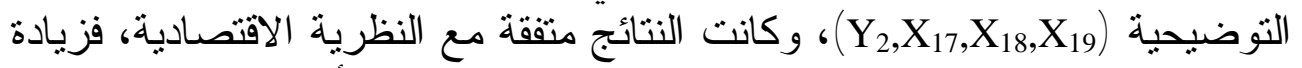

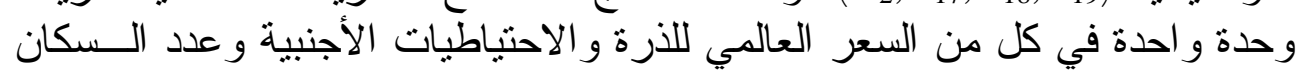

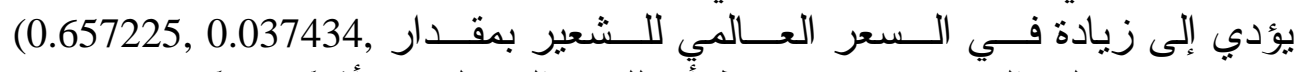

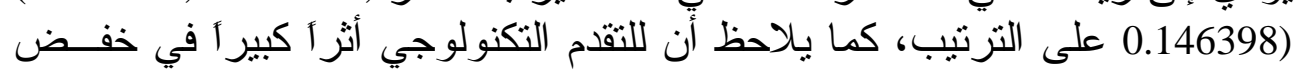

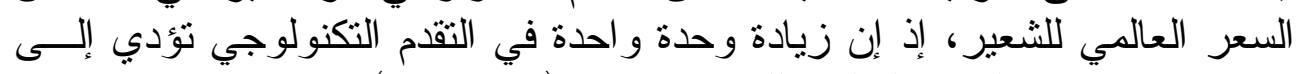

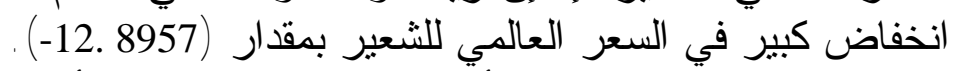

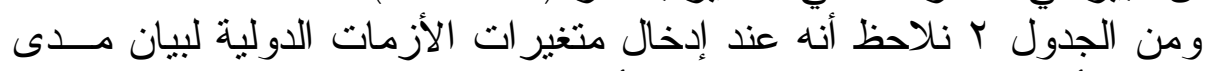

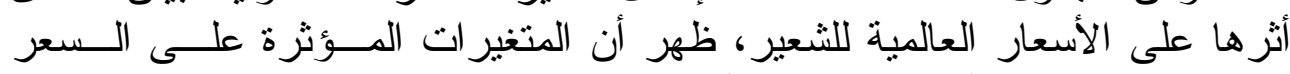

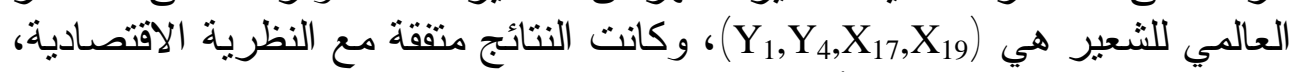

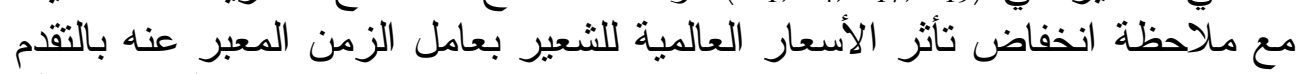

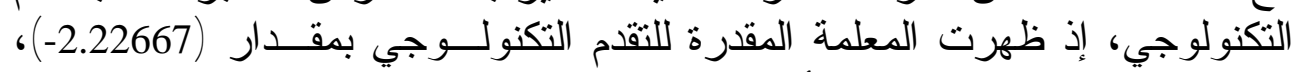

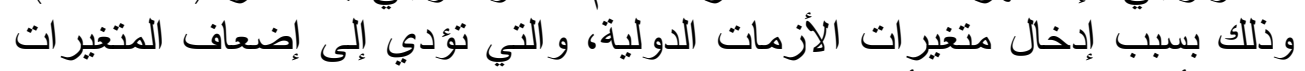
ذات الأثر المباشر في الأسعار العالمية للحبوب. الإل 


\section{يجيى و عبدالله [بra]}

\section{الجدول الإيعار}

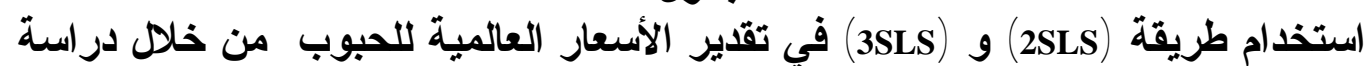

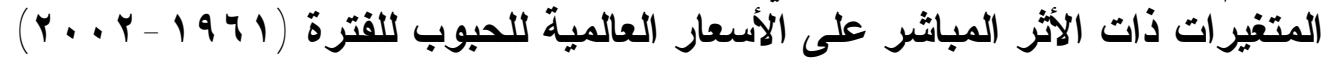

\begin{tabular}{|c|c|c|c|c|c|c|}
\hline Methods & $\begin{array}{c}\text { Dependent } \\
\text { Variable }\end{array}$ & Variables & $\begin{array}{c}\text { Parameter } \\
\text { Estimate }\end{array}$ & $\begin{array}{c}\text { Standard } \\
\text { Error }\end{array}$ & t Value & $\operatorname{Pr}>|t|$ \\
\hline \multirow{17}{*}{ 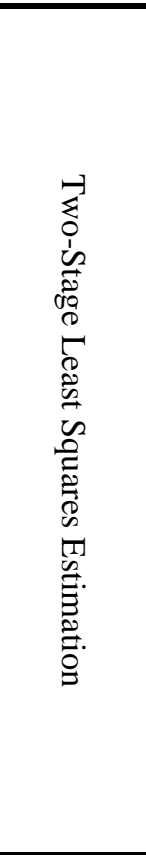 } & \multirow{4}{*}{$\mathrm{Y}_{1}$} & Intercept & -19.666 & 6.092242 & -3.23 & 0.0026 \\
\hline & & $\mathrm{Y}_{2}$ & 0.093838 & 0.039121 & 2.4 & 0.0215 \\
\hline & & $\mathrm{Y}_{3}$ & 0.0989689 & 0.15141 & 6.54 & $<.0001$ \\
\hline & & $\overline{X_{3}}$ & 0.265396 & 0.08632 & 3.07 & 0.0039 \\
\hline & \multirow{4}{*}{$\mathrm{Y}_{2}$} & Intercept & -0.35701 & 10.1276 & -0.04 & 0.9721 \\
\hline & & $\mathrm{Y}_{3}$ & 0.157138 & 0.032654 & 4.81 & $<.0001$ \\
\hline & & $\mathrm{Y}_{4}$ & 0.332044 & 0.182517 & 1.82 & 0.0768 \\
\hline & & $\overline{X_{7}}$ & 0.531599 & 0.146284 & 3.63 & 0.0008 \\
\hline & \multirow{4}{*}{$\mathrm{Y}_{3}$} & Intercept & -128.14 & 93.3611 & -1.37 & 0.1779 \\
\hline & & $\mathrm{Y}_{4}$ & 3.559216 & 1.101307 & 3.23 & 0.0025 \\
\hline & & $X_{1}$. & -13.4113 & 4.34768 & -3.08 & 0.0038 \\
\hline & & $\mathrm{X}_{18}$ & 0.066772 & 0.029561 & 2.26 & 0.0297 \\
\hline & \multirow{5}{*}{$\mathrm{Y}_{4}$} & Intercept & -466.321 & 167.6739 & -2.78 & 0.0085 \\
\hline & & $\mathrm{Y}_{2}$ & 0.634888 & 0.069696 & 9.11 & $<.0001$ \\
\hline & & $\mathrm{X}_{17}$ & 0.042771 & 0.007863 & 5.44 & $<.0001$ \\
\hline & & $\mathrm{X}_{18}$ & 0.167124 & 0.056299 & 2.97 & 0.0052 \\
\hline & & $\mathrm{X}_{19}$ & -14.6876 & 4.297371 & -3.42 & 0.0015 \\
\hline \multirow{17}{*}{ 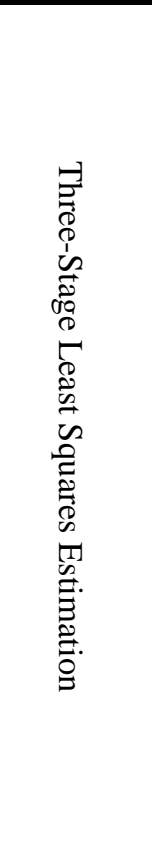 } & \multirow{4}{*}{$\mathrm{Y}_{1}$} & Intercept & -22.341 & 6.033264 & -3.7 & 0.0007 \\
\hline & & $\mathrm{Y}_{2}$ & 1.052445 & 0.036732 & 2.54 & 0.0152 \\
\hline & & $\mathrm{Y}_{3}$ & 0.093441 & 0.143684 & 7.32 & $<.0001$ \\
\hline & & $\mathrm{X}_{3}$ & 0.23142 & 0.083811 & 2.76 & 0.0088 \\
\hline & \multirow{4}{*}{$\mathrm{Y}_{2}$} & Intercept & -7.75254 & 9.902218 & -0.78 & 0.4385 \\
\hline & & $\mathrm{Y}_{3}$ & 0.194737 & 0.029621 & 6.57 & $<.0001$ \\
\hline & & $\mathrm{Y}_{4}$ & 0.355468 & 0.174372 & 2.04 & 0.0485 \\
\hline & & $\mathrm{X}_{7}$ & 0.465007 & 0.139414 & 3.34 & 0.0019 \\
\hline & \multirow{4}{*}{$\mathrm{Y}_{3}$} & Intercept & -178.821 & 88.65678 & -2.02 & 0.0508 \\
\hline & & $\mathrm{Y}_{4}$ & 4.239811 & 1.073581 & 3.95 & 0.0003 \\
\hline & & $\overline{X_{1}}$. & -16.3561 & 3.963733 & -4.13 & 0.0002 \\
\hline & & $\mathrm{X}_{18}$ & 0.075748 & 0.027609 & 2.74 & 0.0092 \\
\hline & \multirow{5}{*}{$\mathrm{Y}_{4}$} & Intercept & -408.514 & 144.3473 & -2.83 & 0.0075 \\
\hline & & $\mathrm{Y}_{2}$ & 0.657225 & 0.065843 & 9.98 & $<.0001$ \\
\hline & & $\mathrm{X}_{17}$ & 0.037434 & 0.006834 & 5.48 & $<.0001$ \\
\hline & & $\mathrm{X}_{18}$ & 0.146398 & 0.048446 & 3.02 & 0.0045 \\
\hline & & $\overline{X_{19}}$ & -12.8957 & 3.724014 & -3.46 & 0.0014 \\
\hline
\end{tabular}




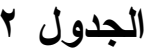

استخذام طريقة (2SLS) و (3SLS) في تقدير الأسعار العالمية للحبوب بوجود ألازمات الاقتصادية للفترة (197)

\begin{tabular}{|c|c|c|c|c|c|c|}
\hline Methods & $\begin{array}{c}\text { Dependent } \\
\text { Variable }\end{array}$ & Variables & $\begin{array}{l}\text { Parameter } \\
\text { Estimate }\end{array}$ & $\begin{array}{c}\text { Standard } \\
\text { Error }\end{array}$ & t Value & $\operatorname{Pr}>|t|$ \\
\hline \multirow{21}{*}{ 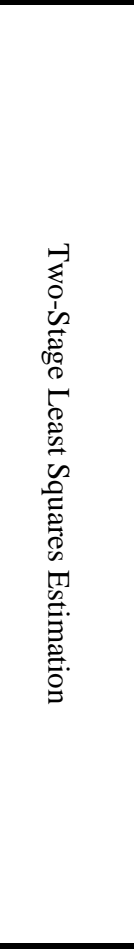 } & \multirow{5}{*}{$\mathrm{Y}_{1}$} & Intercept & -250.11 & 24.27664 & -10.3 & $<.0001$ \\
\hline & & $\overline{Y_{4}}$ & 1.7101 & 0.22308 & 7.67 & $<.0001$ \\
\hline & & $X_{17}$ & -0.12114 & 0.011986 & -10.11 & $<.0001$ \\
\hline & & $\mathrm{X}_{18}$ & 0.066952 & 0.006057 & 11.05 & $<.0001$ \\
\hline & & $X_{23}$ & 19.48987 & 5.043993 & 3.86 & 0.0004 \\
\hline & \multirow{5}{*}{$\mathrm{Y}_{2}$} & Intercept & -39.2162 & 31.46747 & -1.25 & 0.2205 \\
\hline & & $Y_{1}$ & 0.52414 & 0.109935 & 4.77 & $<.0001$ \\
\hline & & $\mathrm{Y}_{4}$ & 0.324176 & 0.255378 & 1.27 & 0.2122 \\
\hline & & $X_{17}$ & -0.028 & 0.01525 & -1.84 & 0.0744 \\
\hline & & $\mathrm{X}_{18}$ & 0.013297 & 0.007926 & 1.68 & 0.1018 \\
\hline & \multirow{6}{*}{$\mathrm{Y}_{3}$} & Intercept & -454.246 & 93.02677 & -4.88 & $<.0001$ \\
\hline & & $\mathrm{Y}_{4}$ & 2.725352 & 0.840928 & 3.24 & 0.0026 \\
\hline & & $\overline{X_{11}}$ & 11.33161 & 5.312034 & 2.13 & 0.0398 \\
\hline & & $X_{17}$ & -0.39283 & 0.067945 & -5.78 & $<.0001$ \\
\hline & & $\mathrm{X}_{18}$ & 0.120564 & 0.022919 & 5.26 & $<.0001$ \\
\hline & & $X_{23}$ & 85.50465 & 19.02014 & 4.5 & $<.0001$ \\
\hline & \multirow{5}{*}{$\mathrm{Y}_{4}$} & Intercept & 33.53921 & 4.15113 & 8.08 & $<.0001$ \\
\hline & & $Y_{1}$ & 0.216198 & 0.127076 & 1.7 & 0.0973 \\
\hline & & $\mathrm{Y}_{2}$ & 0.270879 & 0.185114 & 1.46 & 0.1518 \\
\hline & & $X_{17}$ & 0.053263 & 0.006862 & 7.76 & $<.0001$ \\
\hline & & $\mathrm{X}_{18}$ & -1.98027 & 0.34033 & -5.82 & $<.0001$ \\
\hline \multirow{16}{*}{ 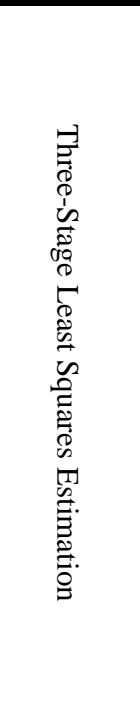 } & \multirow{5}{*}{$\mathrm{Y}_{1}$} & Intercept & -260.093 & 23.74933 & -10.95 & $<.0001$ \\
\hline & & $\mathrm{Y}_{4}$ & 2.023494 & 0.185703 & 10.9 & $<.0001$ \\
\hline & & $X_{17}$ & -0.12511 & 0.011811 & -10.59 & $<.0001$ \\
\hline & & $X_{18}$ & 0.065464 & 0.006034 & 10.85 & $<.0001$ \\
\hline & & $X_{23}$ & 8.639572 & 3.353022 & 2.58 & 0.0141 \\
\hline & \multirow{5}{*}{$\mathrm{Y}_{2}$} & Intercept & -57.936 & 29.76101 & -1.95 & 0.0592 \\
\hline & & $\mathrm{Y}_{1}$ & 0.458098 & 0.103982 & 4.41 & $<.0001$ \\
\hline & & $\mathrm{Y}_{4}$ & 0.491884 & 0.237621 & 2.07 & 0.0455 \\
\hline & & $X_{17}$ & -0.03689 & 0.01446 & -2.55 & 0.015 \\
\hline & & $\mathrm{X}_{18}$ & 0.01754 & 0.007592 & 2.31 & 0.0265 \\
\hline & \multirow{6}{*}{$\mathrm{Y}_{3}$} & Intercept & -461.193 & 92.45403 & -4.99 & $<.0001$ \\
\hline & & $\mathrm{Y}_{4}$ & 3.148021 & 0.825511 & 3.81 & 0.0005 \\
\hline & & $X_{11}$ & 9.765659 & 4.802893 & 2.03 & 0.0498 \\
\hline & & $X_{17}$ & -0.38284 & 0.064564 & -5.93 & $<.0001$ \\
\hline & & $\mathrm{X}_{18}$ & 0.119606 & 0.022874 & 5.23 & $<.0001$ \\
\hline & & $X_{23}$ & 71.18821 & 18.41522 & 3.87 & 0.0004 \\
\hline
\end{tabular}




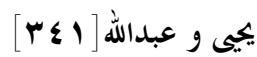

\begin{tabular}{|c|c|c|c|c|c|c|}
\hline Methods & $\begin{array}{c}\text { Dependent } \\
\text { Variable }\end{array}$ & Variables & $\begin{array}{c}\text { Parameter } \\
\text { Estimate }\end{array}$ & $\begin{array}{c}\text { Standard } \\
\text { Error }\end{array}$ & $\mathbf{t}$ Value & $\operatorname{Pr}>|\mathbf{t}|$ \\
\hline \multirow{3}{*}{} & \multirow{3}{*}{$\mathrm{Y}_{4}$} & Intercept & 30.60842 & 3.684919 & 8.31 & $<.0001$ \\
\cline { 3 - 7 } & $\mathrm{Y}_{1}$ & 0.23568 & 0.07841 & 3.01 & 0.0047 \\
\cline { 3 - 7 } & $\mathrm{Y}_{4}$ & 0.311841 & 0.109445 & 2.85 & 0.0071 \\
\cline { 3 - 7 } & & $\mathrm{X}_{17}$ & 0.057009 & 0.006585 & 8.66 & $<.0001$ \\
\cline { 3 - 7 } & & $\mathrm{X}_{19}$ & -2.22667 & 0.325564 & -6.84 & $<.0001$ \\
\hline
\end{tabular}

الاستنتاجات

من أهم الاستتاجات التي تم التوصل إليها:

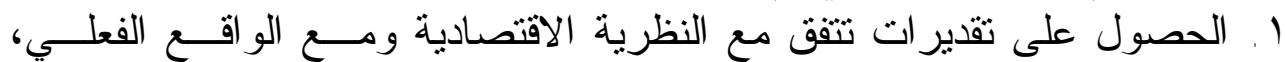
كعززة بالنظرية الإحصائية.

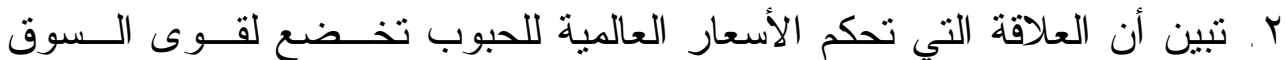

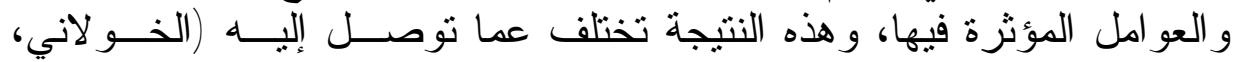

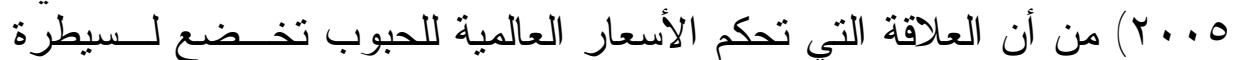

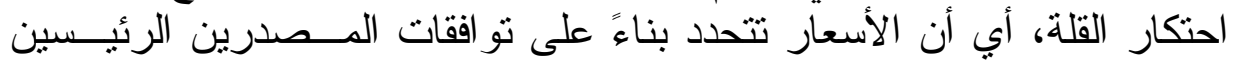

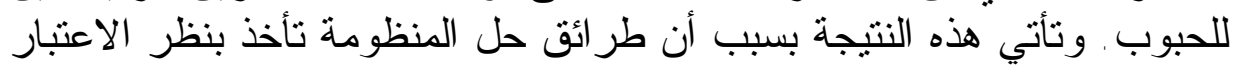

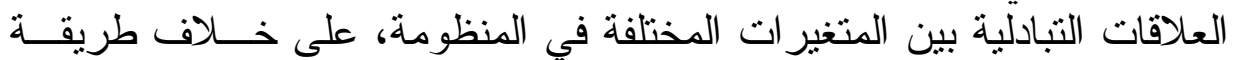

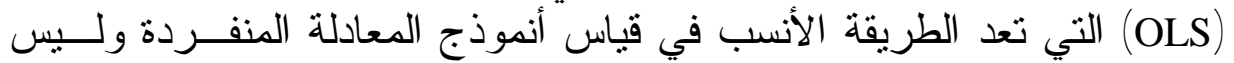

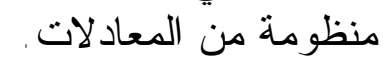

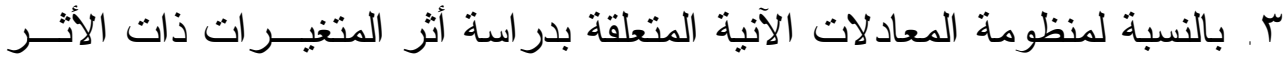

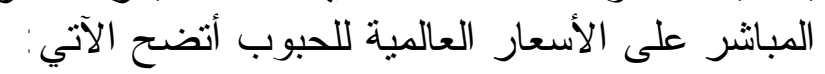

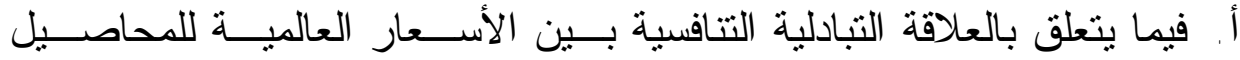

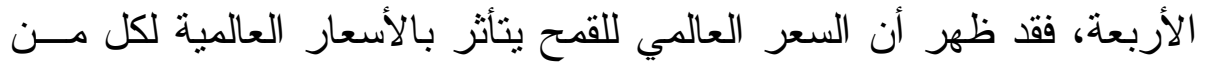

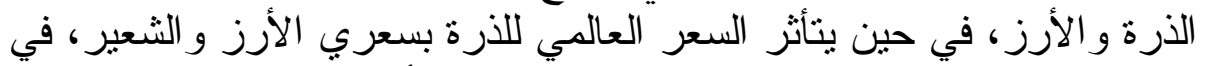

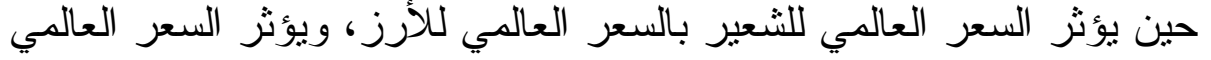

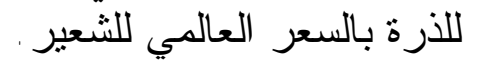

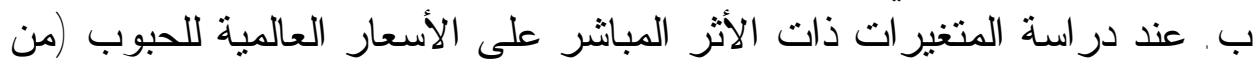

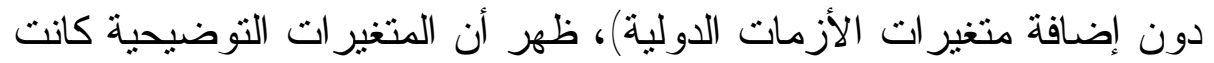

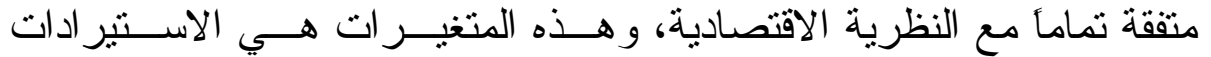

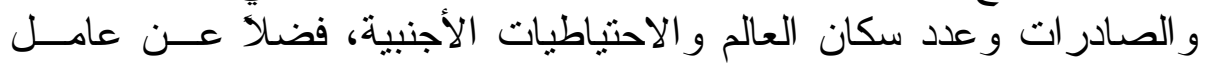

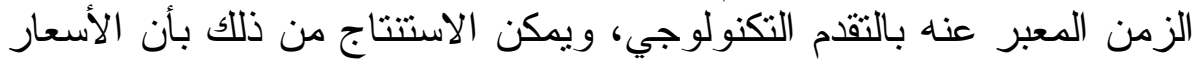

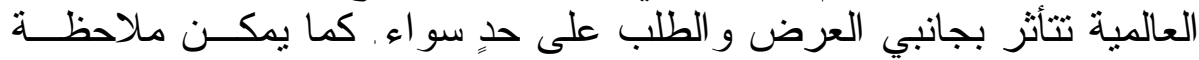

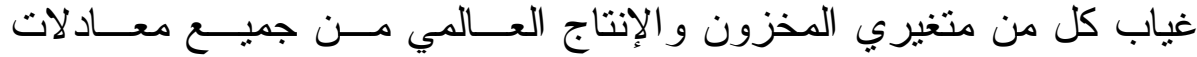

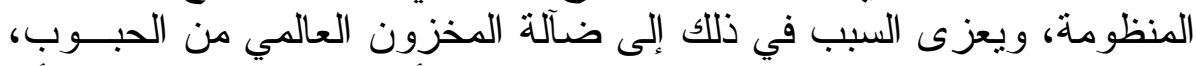
مما يعني عدم مقدرته على إحداث تغييرات في الأسعار العالمية للحبوب، أما لاليا 
معدل الإنتاج العالمي من الحبوب فهو في تناقص مستمر، ذلك للك لأن التطـــور

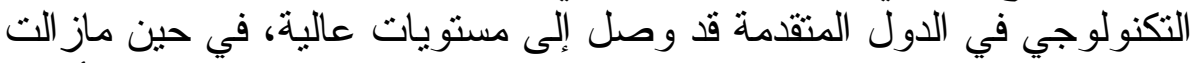

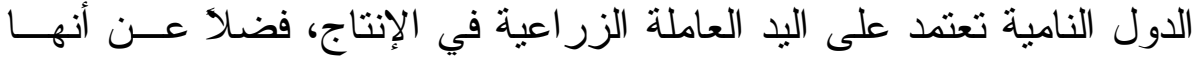

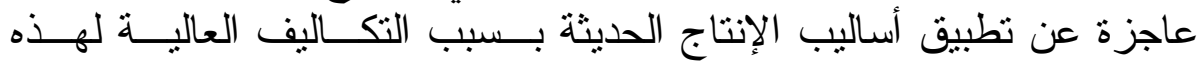

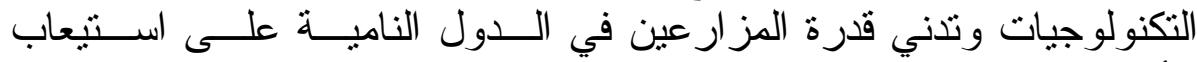
الأساليب الحديثة في الإنتاج.

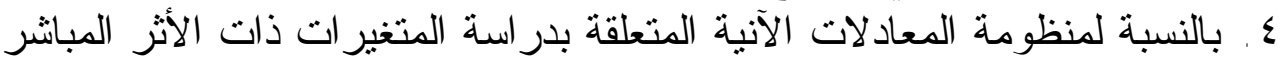

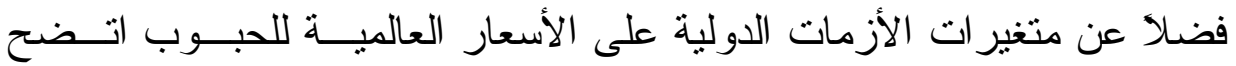
الآتي: أ. فيما يتعلق بالعلاقة التبادلية التتافسية بين الأسعار العالمية للمحاصيل الأربعة

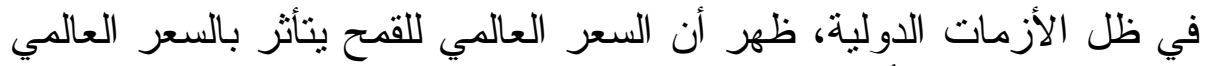

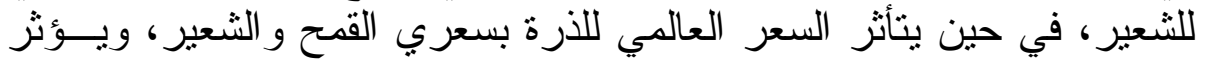

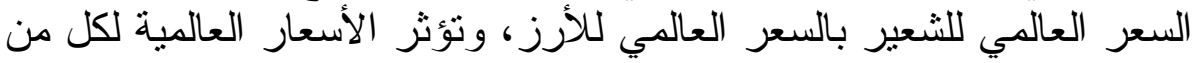

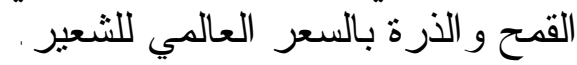

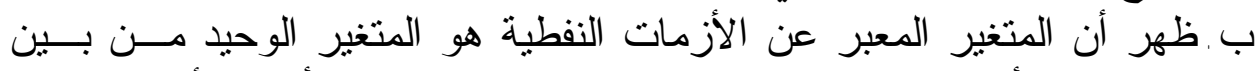

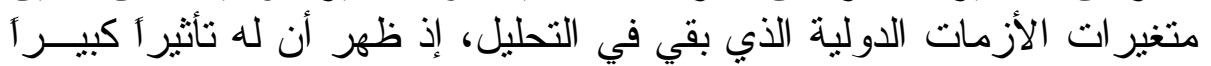

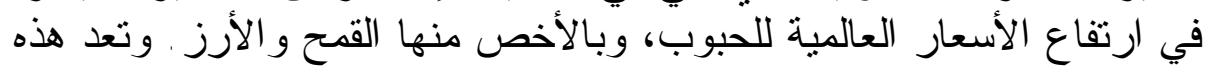

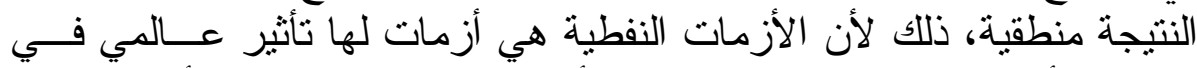

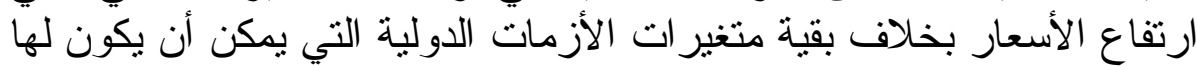

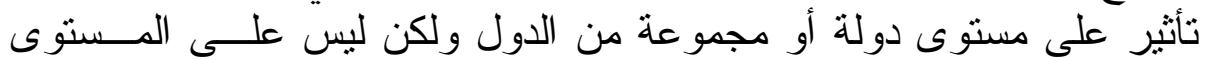

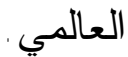

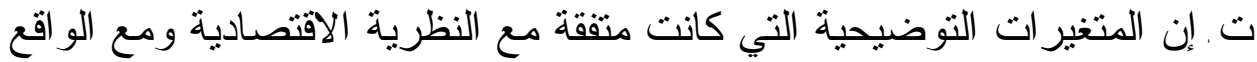

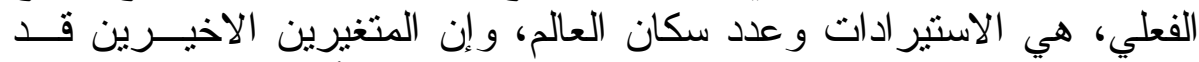

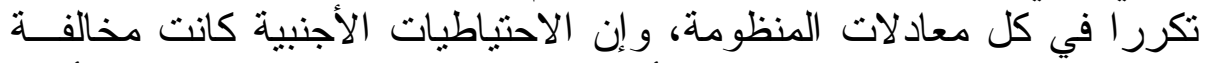

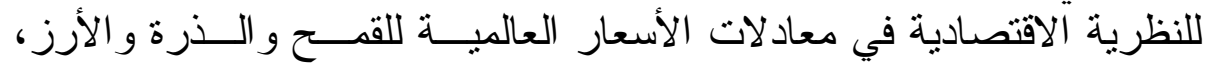

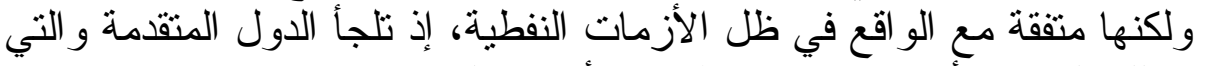

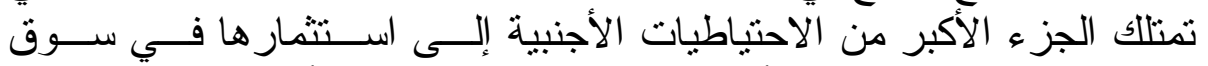

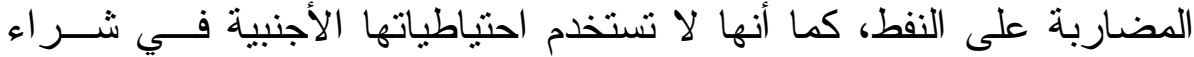

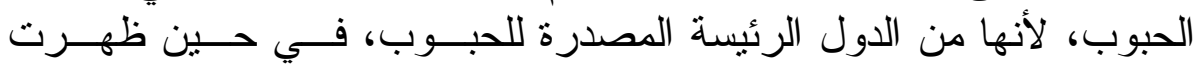

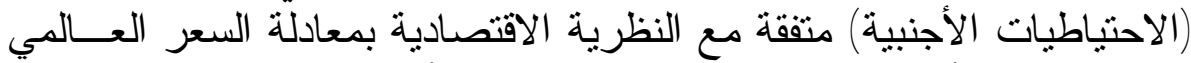

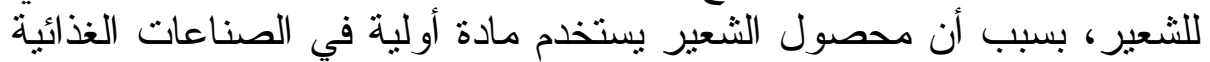

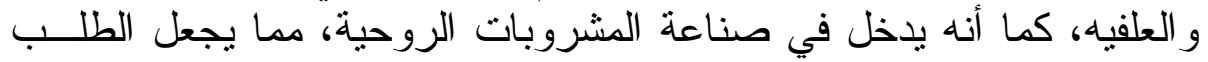
عليه كبير آ و لاسيما في الدول المتقدمة نظر آ لارتفاع المستوى المعانثي ودخل 


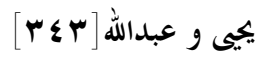

\section{أولاً - المر اجع باللفة العربية}

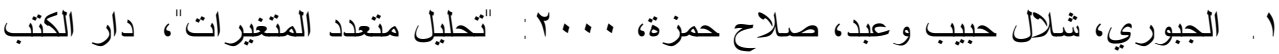

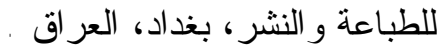

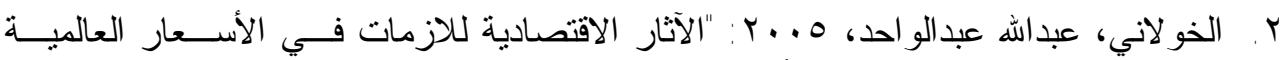

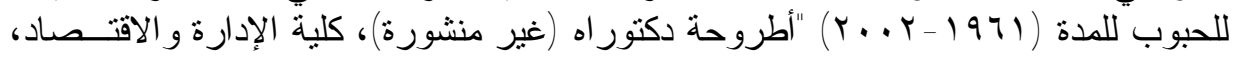

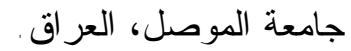
r. عو اد، علاء الدين حسن، 991 (: "القياس الاقتصادي"، الطبعة الأولى، مطابع دار الثرق،

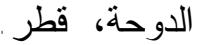

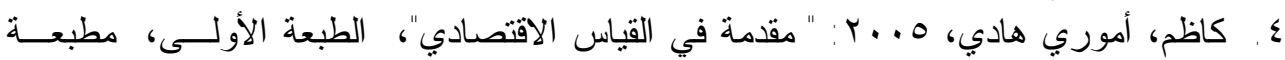

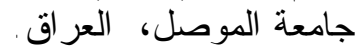

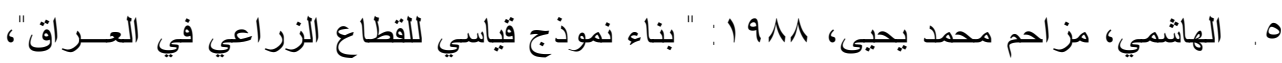
رسالة ماجستير، كلية الإدارة و الاقتصاد، جامعة بغداد، العر اق.

\section{ثانياً - المراجع باللغة الاجنبية}

1. Gujarati,D. N. 1988; "Basic Econometrics", McGraw - Hill Book Company, New York.

2. Intrilligator,M. D. \&Bodkin,R. G. \&Hsiao,C. 1996;"Econometr-ics Models, Techniques and Applications", Prentice Hall. 